\title{
44. ASTRONOMY FROM SPACE
}

PRESIDENT: J.E. Trümper
VICE-PRESIDENT: G.G. Fazio
ORGANIZING COMMITTEE:
B.F. Burke, J.B. Hutchings, E.B. Jenkins (Past President), S.D. Jordan, B.D. Savage, W. Wamsteker

\section{Introduction}

\author{
Joachim E. Trümper
}

The period covered by this report has seen very significant progress in the different fields of space astronomy. In the infrared and submillimeter region the cosmic Background Explorer has continued to dominate the space activities. The most significant result was the first detection of anisotropies in the cosmic background radiation. In the optical and UV region the Hubble Space Telescope, launched in spring 1990 has produced a wealth of significant scientific results despite the image aberrations which are expected to be corrected by the repair mission in December 1993. The astrometric Hipparcos mission reached its scientific goals by producing 1.5 milliarcsec positions and 1 milliarcsec/year proper motions. In the EUV region the fist photometric all-sky survey 1990/91 performed by the Wide Field Camera on ROSAT (Röntgen satellite) has increased the number of EUV sources by almost two orders of magnitude (to 400). A further step was made with the Extreme Ultraviolet Explorer (EUVE launched in 1992) providing increased survey sensitivity and high spectral resolution capabilities. Further spectroscopic data in the UV/EUV were obtained with the UV telescopes on the ASTRO shuttle mission and on the ASTROSPAS, a shuttle attended free flyer. In X-ravs more than 50000 new X-ray sources were discovered by means of the ROSAT all-sky survey in 1990/9l which also led to a much improved knowledge of the galactic and extragalactic soft $X$-ray background. In 1991-93 a few thousand ROSAT pointed observations, including very deep ones, produced a wealth of new results. The Broad Band X-ray Telescope (BBXRT) flown on the ASTRO space shuttle mission in 1990 concentrated on medium resolution X-ray spectroscopy. As the successor of Ginga which reentered in fall 1991 the ASCA satellite (Advanced Satellite for Cosmology and Astrophysics), launched in spring 1993, provides new observational capabilities in imaging X-ray spectroscopy. In hard X-rays the Granat mission allowed to study the time variability of compact $X$-ray sources, in particular the $X$-ray transients.

Gamma-ray astronomy experienced a boost by the launch of the Compton Gamma-ray Observatory in 1991 delivering many new significant results on gamma ray bursts, pulsars, diffuse line emission and quasars.

Last but not least, the most significant event for solar physics from space was the launch and operation of the Yohkoh satellite which yielded a large database for the study of high-energy solar phenomena that accompany solar activity.

All in all the results of these new missions represent a strong stimulus for all fields of astronomy and astrophysics. Two general trends should be mentioned: first the role of coordinated multiwavelength observations involving both space and ground-based observatories is rapidly increasing. There have been a number of very productive campaigns of this kind, such as the RIASS programme (ROSAT-IUE) or the World Astronomy Department. Another important aspect is that space missions yield digitized and calibrated datebases which lend themselves to archiving. The databases of IRAS, IUE, HST, Einstein, EXOSAT, ROSAT etc., as well as the digital optical and radio surveys, have become very powerful tools for astrophysical research. 


\section{Infrared Space Astronomy}

Giovanni G. Fazio

\section{INTRODUCTION}

During the period from 1990 to 1993 the data from the Cosmic Background Explorer (COBE) satellite has continued to dominate the results in space infrared and submilimeter astronomy. The most spectacular result was the first detection of an anisotropy in the cosmic background radiation (CBR). Continued improvements in the accuracy of the measurements of the CBR temperature have shown no deviation from blackbody radiation. Impressive maps of the entire galactic plane were produced at near-infrared wavelengths and in the spectral lines of [CII] and [NII]. Detection of the source F10214+4724 in the Infrared Astronomical Satellite (IRAS) Faint Source Survey data base set off a flurry of exciement and interest. F10214+4724 is a galaxy at a redshift of 2.286 and is the most luminous and massive single source known in the universe. The IRAS database, which has improved with continued analysis, when combined with ground-based and space observations at other wavelengths has produced many new results in the study of starburst galaxies and AGN, infrared luminous galaxies, the origin of elliptical and radio galaxies, protostars, galactic structure, and new views of interstellar dust. The Kuiper Airborne Observatory (KAO) continues to remain a premier facility for far-infrared and submillimeter astronomy. KAO obsrvations have produced important advances in our knowledge of star formation processes, the intersteilar medium, the Galactic center, and stellar and planetary astronomy.

The COBE results are so extensive that they will be treated in a separate section of the report of this Commission. In this section special attention will be given to the IRAS and KAO results, as well as other results from balloon-borne telescopes and rocket observations.

\section{THE INFRARED ASTRONOMICAL SATELLITE (IRAS)}

Follow-up observations of galaxies with bolometric luminosities that are dominated by the far-infrared radiation detected by IRAS continue to constitute a major fraction of the published literature in extragalactic astronomy. A comprehensive review of this wide field of research is beyond the scope of this summary. This is merely an attempt to cover some highlights.

\section{IRAS F10214+4724: Starburst in a Protogalaxy or Dominant AGN?}

Observations of source F10214+4724 in the IRAS Faint Source Survey by Rowan-Robinson et al. (1991) set off a flurry of excitement and interest that has inspired numerous additional observations of this source, as well as searches for others like it. F10214+4724, at a redshift of 2.286 , has an estimated luminosity of $\sim 3 \times 10^{14} \mathrm{~L}_{\odot}$ and a mass of $\sim 3 \times 10^{11} \mathrm{~L}_{\odot}$, making it the most massive and luminous single source known in the universe. The look-back time corresponds to approximately $80 \%$ of the age of the universe. The high excitation emission lines in the spectrum observed by Rowan-Robinson et al. (1991) indicate the presence of an AGN, but whether or not the source represents a protogalaxy undergoing its first burst of star formation, or a dust enshrouded AGN, is still up for debate. The mass of neutral molecular hydrogen was estimated soon after discovery of the galaxy, with the amazing detection of the CO 3-2 transition, first by Brown \& vanden Bout (1991), and then confirmed by others (e.g., Solomon, Radford, \& Downes 1992). These observations showed that F10214+4724 has an infrared to CO luminosity ratio twice that of most IR-luminous galaxies and about 30 times higher than that of normal spirals. Its IR colors and high IR/CO luminosity ratio indicate that F10214+4724 is similar to nearby ultraluminous galaxies that are known to be mergers. This galaxy is a primeval molecular galaxy with the mass of a large spiral, but with most of the mass in molecular gas rather than stars. It is the best candidate yet discovered for a forming disk galaxy or cluster of galaxies. 
One reason for the great interest in F10214+4724, as for lower redshift ultraluminous infrared galaxies, concerns the dominant source of the extremely high far-infrared luminosity detected by IRAS. There is little doubt that both a luminous starburst and a non-stellar active nucleus are present in the source. The interesting question is- which component dominates the energy budget for the tremendous $10^{14}$ solar luminosities that this source generates? There is abundant evidence that F10214+4724 is a primeval galaxy undergoing rigorous star formation, including $\sim 6 \times 10^{11} \mathrm{M}_{\odot}$ of molecular gas (Solomon et al. 1992), and a UV-to-radio continuum energy distribution which is most simply interpreted as a powerful starburst (Rowan-Robinson et al. 1993). Likewise, there is substantial evidence for an embedded AGN: high excitation emission lines (Rowan-Robinson et al. 1991), strong polarization (Lawrence et al. 1993), and a very compact morphology in the light of the $\mathrm{H} \alpha$ emission line (Soifer et al. 1992). New results show [NII]/H $\alpha$ and $[\mathrm{OIII}] / \mathrm{H} \beta$ emission line ratios to be typical of those found in type 2 Seyfert galaxies (Elston et al. 1993). High spatial resolution near-infrared (rest-frame optical) imaging shows a double structure in the central 2 arcsec which suggests an interacting/merging system of two or more galaxies (Soifer et al. 1992). The contention is that this source may be a high redshift analog to the ultraluminous infrared galaxies observed in the local universe. Continuing studies of this exciting object will undoubtedly have a great impact on our understanding of galaxy formation and cosmology.

\section{The Source of the Far-Infrared Emission in Stäburst Galaxies and AGN}

An important work which addresses the issue of the source of the far-infrared emission in active galaxies was published by Mouri \& Taniguchi (1992). Using observations of [O I] $\lambda 6300, \mathrm{H} \alpha, \mathrm{H}_{2} \mathrm{~S}(1)$, and $\mathrm{Br} \gamma$ emission lines in relation to the $25 \mu \mathrm{m}$ to $60 \mu \mathrm{m}$ flux ratios observed by IRAS, they constructed a useful model in which the evolutionary stage of starburst and AGN activity determines the relevant extent to which various sources of far-infrared radiation contribute to the total luminosity measured by IRAS. The far-infrared radiation emitted from AGNs is thus a mixture of a flat nonthermal component from the AGN and a steep thermal component from dust heated by young stars, with their fraction varying from one source to another, and likely changing in time as the various components evolve. Although more complex than a more simplistic model we might hope for, this result is likely a more realistic treatment of the complexities involved in interpreting the far-infrared emission from F10214+4724 and other sources which have evidence for AGNs and starbursts that coexist in the same galaxy.

\section{Superwinds}

A principal result to emerge from ground-based follow-up observations of infrared galaxies selected using IRAS color ratios is that many starburst galaxies have large-scale nebulae of ionized gas which often have the morphology and kinematics of a bi-polar outflow many kiloparsecs in size. In these "superwinds," the kinetic energy provided by supernovae and winds from massive stars in a central starburst drives a large-scale outflow that can shock heat and accelerate ambient interstellar and intergalactic gas. This work, pioneered by Heckman and his collaborators (e.g., Heckman, Armus, \& Miley 1990; Armus, Heckman, \& Miley 1990) demonstrates that the evolution of the interstellar medium, as well as the intergalactic medium, is profoundly affected by large-scale outflows on a galaxy-wide scale. The superwind process may also play a critical role in driving out much of the gas and dust associated with a starburst, diminishing the extinction at optical and ultraviolet wavelengths and thus allowing a previously embedded ultraviolet-excess AGN to dominate the bolometric luminosity of the source. This has important implications for current theories of the formation and evolution of classical quasars, radio galaxies and elliptical galaxies.

\section{The Origin of Elliptical Galaxies}

A long-standing argument against the hypothesis that most massive elliptical galaxies form primarily by merging spiral galaxies is the extremely high mass densities in the cores of the former compared to the later. A recent study of infrared ultraluminous galaxies (those with infrared luminosities comparable to classical quasars, $\mathrm{L}_{\mathrm{ir}}>10^{12} \mathrm{~L}_{\odot}$ ) suggests that these objects are elliptical galaxies in the process of formation (Kormendy, \& Sanders 1992). The typical central densities of molecular gas, $\sim 100 \mathrm{M}_{\odot} \mathrm{pc}^{-3}$, are as high as the stellar mass densities in elliptical galaxies, and the intense starbursts taking place in these sources 
are just what is expected during the dissipative collapse induced by a merger. The high gas density is transformed into a high stellar mass density, and the high metallicities of ellipticals are a natural outcome, since even the oldest ellipticals would form from starburst-enriched gas. These results have implications for searches for protogalaxies at high redshift because forming elliptical galaxies may be heavily enshrouded by dust and hence be best detected at infrared wavelengths.

\section{The Origin of Radio Galaxies}

One of the many early discoveries made by IRAS is that a significant fraction of radio-loud galaxies are luminous far-infrared sources (Golombek, Miley, \& Neugebauer 1988). Radio galaxies have traditionally been thought to be giant ellipticals which are devoid of extensive quantities of gas and dust. However, a new $\mathrm{CO}(\mathrm{J}=1 \rightarrow 0)$ line survey of powerful radio-selected galaxies with $\log \mathrm{P}_{408 \mathrm{MHz}}>23.5 \mathrm{~W} \mathrm{~Hz}^{-1}$ has doubled the number of previously known $\mathrm{CO}$ detections of powerful radio galaxies (Mazzarella, Graham, Sanders, \& Djorgovski 1993). The computed $\mathrm{H}_{2}$ masses range from $\sim 3 \times 10^{9} \kappa_{i} \sim 2 \times 10^{10} \mathrm{M}_{\odot}$, simil : to the $\mathrm{H}_{2}$ masses derived for other luminous infrared galaxies, but in stark contrast to the low $\mathrm{H}_{2}$ gas masses found in radio-quiet IRAS-selected elliptical galaxies, $10^{7}-10^{8} M_{\odot}$ (Lees, Knapp, Rupen, \& Phillips 1990). The results suggest that rich supplies of molecular gas may be ubiquitous in powerful radio-selected galaxies detected by IRAS, and the origin of powerful radio galaxies may be closely related to the genesis of dust enshrouded quasars and classical UV-excess quasars through the merging of gas-rich disk galaxies. The recent detection of weak, large-scale radio lobes associated with the eastern nucleus of the merging galaxy pair Markarian 463 bolsters the case for a unified model for the origin of quasars and powerful radio galaxies via an infrared-luminous phase (Mazzarella et al. 1991). The radio source in Mrk $463 \mathrm{E}$ is $20 \mathrm{kpc}$ in extent, the largest known to be associated with a gas-rich spiral galaxy. Mrk 463 may exemplify a "missing link" or transition case between the confined ( $<$ few $\mathrm{kpc}$ ) linear radio sources observed in Seyfert galaxies and the extended (hundreds of $\mathrm{kpc}$ ) sources in classical quasars and radio galaxies.

\section{Embedded AGNs in Infrared-Luminous Galaxies}

Near-infrared spectroscopy provides a useful way to take advantage of the decreased extinction due to dust compared to optical wavelengths; the extinction, in magnitudes, at $2 \mu \mathrm{m}$ is about one tenth that in the visual band. Using this tool, a number of workers have searched for evidence of embedded broad-line regions in infrared-luminous galaxies. Such sources have been found in the galaxies IRAS 20460+1925, IRAS $23060+0505$ (Hines 1991), and IRAS 20460-1447 (Nakajima et al. 1991). This work is important because it demonstrates that there is a population of obscured QSO's, and that they can be selected by their warm infrared energy distributions and QSO-like luminosities. Nevertheless, a recent comprehensive study of 14 ultraluminous infrared galaxies by Goldader et al. (1993) found no new (embedded) broad-line regions that were not known from previous optical spectroscopy. Emission lines from present and past vigorous star formation dominate the infrared spectra of these galaxies. These results place important constraints on unified models which maintain that galaxies with classical type 2 Seyfert spectra may harbor type 1 Seyfert nuclei, but they are invisible because of obscuration due to dust.

A parallel effort to detect embedded AGNs in infrared-luminous galaxies took place using very long baseline interferometry at radio wavelengths (Lonsdale, Smith, \& Lonsdale 1993). Over half of a sample of 31 infraredluminous galaxies show high-brightness temperature emission, with $\mathrm{T}_{\mathrm{b}}>10^{5} \mathrm{~K}$ and structure on scales of 5-150 milliarcseconds. The limits for non-detected sources are similar, consistent with the hypothesis that most of these galaxies have compact AGN cores at a level of a few percent of the total radio flux density. Structural information and energy budget considerations rule out a single supernova interpretation of the compact radio emission in these galaxies, although the possibility of several simultaneous, extraordinarily luminous radio supernovae within the central few hundred cubic parsec cannot be ruled out. Nevertheless, these results provide important new evidence for AGNs obscured by dust in a surrounding starburst. Whether or not the embedded AGNs dominate over radiation from hot stars in the total energy deposited into heating the dust observed in the far-infrared remains an active topic of research. 


\section{IRAS Protostars}

IRAS showed that stars of roughly solar mass form out of clumps of molecular material ranging in mass and size from 1-100 $\mathrm{M}_{\odot}$ and $0.02-0.2 \mathrm{pc}$. The temperature and density of these clumps have values around 15-25 $\mathrm{K}$ and $10^{4} \mathrm{~cm}^{-3}$ (Beichman et al. 1986; Myers et al. 1987). The 1-100 $\mu \mathrm{m}$ spectral energy distributions (SED's) require a hot central star $\left(\mathrm{T} \sim 3000 \mathrm{~K}\right.$ ), many magnitudes $\left(\mathrm{A}_{V} \sim 10-50 \mathrm{mag}\right)$ of intervening dust to absorb and re-radiate the stellar radiation, and a massive disk $\left(\sim 0.1 \mathrm{M}_{\odot}\right)$ of material located within a relatively empty, 1 $100 \mathrm{AU}$, central void around the star (Myers et al. 1987). These early observational samples have been expanded to include hundreds of young stars with multi-wavelength studies of the Taurus (Kenyon et al. 1990; Beichman, Boulanger and Moshir 1992), Corona Australis (Wilking et al. 1992), Ophiuchus (Wilking, Lada and Young 1989) and the Chamaeleon clouds (Prusti, Whittet and Wesselius 1991; Gauvin and Strom 1992).

A combination of IRAS and ground-based observations of T Tauri stars strengthens the case for the existence of disks around many of these objects. Roughly $80 \%$ of $\mathrm{T}$ Tauri stars have passively heated disks contributing $\sim 20 \%$ or less of the total luminosity of the system (Rucinski 1985; Strom et al. 1988; Cohen, Emerson and Beichman 1989). The remaining $20 \%$ of the stars have substantial infrared excesses, suggesting the existence of active disks powered by accretion with infall rates as high as $10^{-5} \mathrm{M}_{\odot} \mathrm{yr}^{-1}$. Hildebrand et al. (1992) have shown that a star+accretion disk model can also satisfy the 1-1000 $\mu \mathrm{m}$ observations of Herbig $\mathrm{Ae} / \mathrm{Be}$ stars, but with mass accretion rates, the disk masses and disk radii that are proportionately larger.

Estimates of disk masses for $\mathrm{T}$ Tauri stars range from 0.001-0.1 $\mathrm{M}_{\odot}$ (Beckwith et al. 1990; Adams, Emerson and Fuller 1990), but are uncertain because of uncertainties in the mass absorption coefficient at long wavelengths. Additional support for the existence of massive disks comes from millimeter observations that show material in Keplerian rotation around the HL Tau (Sargent and Beckwith 1987, 1991), and a resolved disk around L1551-IRS-5 (Keene and Masson 1990) implying disk masses $\sim 0.1 \mathrm{M}_{\odot}$. The existence of a large amount of molecular gas and dust for a few million years has obvious implications for the formation of planetary systems (Strom et al. 1989; Keene and Masson 1990). Ground-based $10 \mu \mathrm{m}$ observations of selected $\mathrm{T}$ Tauri stars set limits on the disk lifetimes in the range 1 to 3 million years (Strom et al. 1989; Skrutskie et al. 1990).

\section{IRAS Luminosity Functions}

The existence of samples of many tens of sources towards a variety of clouds permits a preliminary comparative analysis of the protostellar luminosity function under various physical conditions. The dark clouds such as Taurus, L1641 and Chamaeleon I share similar luminosity functions marked by a relatively small number of objects of intermediate luminosity. (Gauvin and Strom 1992; Strom and Strom 1991; Kenyon et al. 1989; Beichman et al. 1992. This observation presents a difficulty for simple accretion models which predict the existence of more high luminosity objects. A natural explanation for the observed excess of low luminosity objects is that infall occurs not onto the central star, but is held in the reservoir of a disk, to be released at a slower rate, and at a lower luminosity over the 1-3 million year lifetime of the disk (Kenyon et al. 1989; Beichman et al. 1992).

The luminosity function of the relatively diffuse dark clouds mentioned above differs from that in more concentrated clouds such as $\rho$ Ophiuchus and $\mathrm{Cr}$. These compact, dense clouds have a greater proportion of moderate and high luminosity $\left(>5 \mathrm{~L}_{\odot}\right.$ ) Class I objects relative to Class II objects (Kenyon et al. 1990; see Zinnecker McCaughrean and Wilking, 1992, for a review). Possible explanations for the differences include a higher accretion rate or a formation of stars of different average mass, $2-3 \mathrm{M}_{\odot}$, in the denser clouds vs. $1-2 M_{\odot}$ in the more diffuse clouds.

\section{Galactic Structure Revealed by IRAS}

The luminous carbon stars and mass-losing Mira variables detected by IRAS provide a nearly extinction free probe of Galactic structure that can be used out to distances beyond the Galactic Center. Analysis of IRAS source counts has shown that the Galaxy has a prominent warp similar to that seen in $21 \mathrm{~cm}$ maps 
(Djorgovski and Sosin 1989). A key feature of the IRAS result is that the progenitors of these stars are intermediate and low mass stars many billions of years old, implying that the warp is not a transient feature of the interstellar medium, but a long-lived feature of the Galaxy. Gravitational perturbations induced by a massive dark halo can produce warps comparable to that seen by IRAS.

IRAS sources selected on the basis of their color or variability to be AGB stars clearly delineate a central concentration of stars toward the Galactic Center. Some authors attribute to a prominent Galactic Bulge (Habing et al. 1985; Rowan-Robinson and Chester 1987; Van der Veen and Habing 1990) while Weinberg (1992a) claims that the counts can be explained with only an axisymmetric exponential disk population. Detailed analysis of COBE results will play an important role in determining whether a spheroidal bulge really exists. Weinberg (1992b) also suggests that the Milky Way is a barred spiral galaxy with arms originating at the termination of a $5 \mathrm{kpc}$ bar defined by variable, mass-losing giants. The contrast in the bar is high, about a factor of 7 in the counts between the minimum and maximum regions at a given radius. The bar seen in the IRAS counts may be related to the triaxial feazure inferred from analysis of $2 \mu 1 n$ ground-based observations (Blitz and Spergel 1991).

\section{New Views of Interstellar Dust}

Analysis of IRAS data have fundamentally changed our view of the interstellar medium. Instead of gas sprinkled with simple $0.1 \mu \mathrm{m}$ silicate and graphite grains, IRAS has shown that there is a continuum between the atoms and molecules in the gas phase, macro-molecules, small dust grains and ultimately large classical grains. Variations in the relative abundances of these species trace directly the strength and hardness of the interstellar radiation field (ISRF) as well as grain formation and destruction processes.

The physical link between $12 \mu \mathrm{m}$ emission and PAHs was greatly strengthened by the observation that the galactic distribution of the $3.3 \mu \mathrm{m}$ PAH feature closely follows that of the $12 \mu \mathrm{m}$ radiation found by IRAS (Girard et al. 1989). The new IRAS Sky Survey Atlas offers a three to five-fold improvement in sensitivity compared to earlier IRAS images at 12 and $25 \mu \mathrm{m}$ and will facilitate study of this phenomena in a variety of environments.

Observations of the $12 \mu \mathrm{m}$ radiation from other galaxies can also be used to learn about small grain physics. The large to small grain abundance ratio is relatively constant in normal galaxies of similar metallicity with a dispersion of less than $40 \%$ (Helou 1986; Helou, Ryter and Soifer 1991). Most of the variation in the $f_{\nu}(12 \mu \mathrm{m}) /\left(f_{\nu}(60 \mu \mathrm{m})+f_{\nu}(100 \mu \mathrm{m})\right)$ ratio is attributed to variations in the interstellar radiation fields in these galaxies which, at increasing levels, destroys small grains and gradually increases the contribution from classical large grains.

A number of groups have addressed the composition of interstellar dust using IRAS data (see Puget and éger 1989 for a review). Clues to the nature of the small grains have come from variations in the strength of $12 \mu \mathrm{m}$ emission as a function of interstellar radiation field (ISRF) strength within molecular clouds and HII regions (Boulanger et al. 1988). A comprehensive model of interstellar dust has been developed that fits the optical and UV extinction curves, the IRAS emission properties, and the longer wavelength submillimeter emission (Désert et al. 1990). The model invokes large grains, small ( $\leq 0.01 \mu \mathrm{m})$ 3-dimensional grains, and smaller $(\sim 10 \AA)$ planar grains (PAHs). Together the grains and PAHs may comprise more than $40 \%$ of all interstellar carbon.

\section{RESULTS FROM NEAR-SPACE OBSERVATORIES}

The Kuiper Airborne Observatory (KAO), a 91-cm telescope mounted in a converted C-141 aircraft capable of reaching altitudes of $12.8 \mathrm{~km}$, continues to remain a premier facility for infrared and submillimeter astronomy. Important advances in our knowledge of star formation processes, the interstellar medium, the Galactic center, and stellar and planetary astronomy have resulted from $\mathrm{KAO}$ observations. 


\section{Molecular Clouds and Star Formation}

Far-infrared polarimetry has led to the first mapping of magnetic fields in dense cloud cores (Hildebrand et al. 1993). Regions of uniform or smoothly varying polarization seen in many clouds indicate highly structured fields despite the presence of turbulence and fragmentation.

Starburst galaxies like NGC 253 and NGC 3256 have enormous rates of massive star formation occuring in their central, obscured regions. KAO observations of infrared fine structure lines (Carral et al. 1993) reveal a highly pressurized interstellar medium populated with numerous, small, dense, molecular clouds. The clouds and HII regions appear to be in pressure equilibrium with a supernova-shocked, hot, low density, all pervasive medium. A feedback mechanism may be indicated in which the pressure generated by the supernovae compresses the molecular clouds and triggers further massive star formation.

KAO maps of a number of pre-main-sequence stars suggest that much of the far-infrared radiation from young stellar objects does not arise from dust in a compact disk, but, in fact, from much more extened circumstellar clouds (Butner et al. 1990; Natta et al. 1992; Natta et al. 1993). In some cases the temperature distribution in these extended clouds suggests possible heating by a central disk rather than by a stellar photosphere.

\section{Interstellar Medium}

KAO maps of several galaxies in the 158 micron [CII] line using an imaging Fabry-Perot spectrometer have been made, leading to the first direct determination of the pressure in the cold atomic medium of external galaxies (Madden et al. 1993). Similar observations in a sample of gas rich galaxies have shown that warm dense gas may represent a substantial fraction of the total gas mass in the nuclear regions of galaxies (Stacey et al. 1991).

\section{Galactic Center}

Images of the [CII] line (Poglitsch, et al. 1991) and of the [OIII] fine structure lines, in conjuction with spectra of far-infrared lines from [OI], [CII], [SiII], [SIII], [NIII], and [OIII] and the 20 to 160 micron continuum at a number of positions both along and across the thermal arched filaments (Erickson et al. 1991 and Colgan et al. 1993) revealed that the dominating ionization mechanism for the thermal filaments in the Galactic Center Radio Arc is consistent with photoionization by local, young massive stars. Contrary to expectations, far-infrared polarization measurements indicate that the magnetic field in the thermal filaments just north of the dust ring is orthogonal to the field in the long non-thermal arcs that traverse and interact with the filaments. The field smoothly follows the curve of the filaments. (Morris et al. 1992).

Observations of the 63 micron [OI] fine structure line toward the SgrA West region (Jackson et al. 1993) showed a large amount of neutral gas in the cavity inside the circumnuclear molecular ring, possibly indicating an infall of gas toward the massive central object SgrA*. Measurements of the far-infrared lines of [SIII], [FeIII], [OIII], [NIII], and [NII] in Sgr A West are all consistent with photoionization by stars with an effective temperature of $35,000 \mathrm{~K}$ and do not require shocks or stellar winds for their production. The densities determined from the [OIII] and [SIII] lines suggest that the doubly ionized lines arise predominantly in the low density gas enclosing the high density 'mini-spiral' seen in radio continuum maps and lower excitation lines such as NeII (Erickson et al. 1993).

Far-infrared polarization measurements have shown that the magnetic field in the dust ring at the center of the Galaxy has the structure expected for a magnetic accretion disk in which angular momentum is removed centrifugally. This is the oniy direct observation of the magnetic field in any accretion disk. A streamer with a longitudinal field penetrates the northern portion of the ring. 


\section{Stellar Astronomy}

Far-infrared spectroscopy of the [FeII] and [NiII] lines from SN 1987A at several epochs have probed the mantle of the supernova (Erickson et al. 1988; Haas et al. 1990, and Colgan et al. 1993). These observations provide evidence for mixing and clumping in SN 1987A, imply a spatial association between the dust clumps and the iron-group elements, and suggest segregation of the iron-group elements from the lighter elements such as $\mathrm{Mg}$ and $\mathrm{O}$. A mysterious high-velocity feature at $3900 \mathrm{~km} / \mathrm{s}$ was also found.

KAO observations of Nova Cygni 1992 (Nova V1974 Cygni) in May, 1992 resulted in identification of a number of atomic lines of hydrogen and neon (Gehrz et al. 1993). Observations in July 1993, showed that the original atomic lines had disappeared and been replaced by a Ne VI line. No infrared continuum was seen in any of the spectra, indicating that dust had not yet condensed from the materials ejected by the nova blast.

Far-infrared [OI] and [SiII] lines have been observed in the oxygen-rich, M2 Iab supergiant, alpha Orionis. Their fluxes are consistent with emission arising from dense, warm gas in the star's inner envelope and implies that nearly all of the available $\mathrm{O}$ and $\mathrm{Si}$ are in atomic form. This is the first detection of far-infrared, fine structure emission from the inner or transition region of a circumstellar envelope, where molecules and dust are expected to form.

\section{Planetary Astronomy}

A combination of laboratory measurements of $\mathrm{H}_{2} \mathrm{O}$ trapped in $\mathrm{SO}_{2}$ matrices, and $\mathrm{KAO}$ spectra of Jupiter's satellite Io, resulted in the discovery of small amounts of water on Io's surface (Salama et al. 1990 and 1993). This demonstrates that hydrogen is present there, and has important implications for our understanding of the chemistry on this satellite.

\section{Balloon-Borne Astronomical Observations}

The 1-meter balloon-borne telescope of the Tata Institute for Fundamental Research continues to produce high resolution far-infrared maps of H II regions including G351.6-1.3/G351.7-1.2, W31, and the galaxies NGC 4945, and the Circinus galaxy (Gosh et al. 1990a; Gosh et al. 1990b; Gosh et al. 1992).

Far-infrared observations of the Cygnus- $X$ region in the galaxy were performed with a $60-\mathrm{cm}$ diameter balloon-borne telescope developed at ETH Zurich in collaboration with the Geneva Observatory. Successful measurements were made on the dust emission and temperature of the molecular cloud complexes DR21/W75N and S106 (Holenstein et al. 1992).

The first large scale far-infrared [CII] line emission maps of the galactic plane were completed using balloonborne telescopes equiped with Fabry-Perot spectrometers by a joint group from the Institute of Space and Astronautical Science (ISAS), Japan, the University of Arizona, and the Kyoto University, Japan (Shibai, H. et al. 1991). Extended, bright [CII] emission throughout the Galaxy. Possible candidates for this emission are extended photodissociation regions heated by the general interstellar radiation field, or extended low-density $\mathrm{H}$ II regions ionized also by the interstellar radiation field.

\section{FUTURE MISSIONS}

The 1990's will see an enormous increase in space activities for infrared and submillimeter astronomy. Some of the major missions planned for the future are described below.

\section{Infrared Space Observatory (ISO)}

ISO is a $60-\mathrm{cm}$ helium-cooled telescope with four focal plane instruments: a short wavelength camera, a multiwavelength photometer/polarimeter, and short and long wavelength spectrometers, covering the spectral 
region from 3 to 200 microns. ISO is an intermediate mission between IRAS and SIRTF in both schedule and capability. It will be launched by ESA in 1995 and will provide the first follow-up on the IRAS results and define further scientific questions for the later SIRTF mission.

\section{Infrared Telescope in Space (IRTS)}

IRTS is a helium-cooled telescope planned for launch in 1995 by Japan's Institute of Space and Astronautical Science. It will carry four experiments: a Fabry-Perot interferometer to map the C II and O I emission from the Galaxy, a submillimeter wavelength radiometer and a near-infrared photometer to measure the extragalactic background radiation, and a mid-infrared spectrometer. Because it shares a platform with other space experiments IRTS will obtain data for only 2 weeks.

\section{Submillimeter-Wave Astronomy Satellito (SWAS)}

SWAS is one of NASA's Small Explorer Class satellites, consisting of a 55-cm ambient temperature telescope with passively cooled detectors, to search for single lines of $\mathrm{H}_{2} \mathrm{O}, \mathrm{O}_{2}, \mathrm{C} \mathrm{I}$, and ${ }^{13} \mathrm{CO}$ in molecular clouds and to qualify several critical submillimeter wave receiver and spectrometer elements to be used on later missions. The launch date is 1995.

The Near Infrared Camera and Multi-Object Spectrometer for the Hubble Telescope (NICMOS)

NICMOS is a second generation NASA instrument for the Hubble Space Telescope that is designed to provide imaging and spectroscopic observations at wavelengths between 0.8 and 3.0 microns. NICMOS contains cryogenically-cooled cameras and spectrometers to cover a wide range of scientific objectives, and has an expected lifetime of grcater than five years.

\section{Space Infrared Telescope Facility (SIRTF)}

SIRTF, one of NASA's Great Observatories, is a 0.85-meter diameter cryogenically-cooled telescope which will be located in a solar orbit for a period of at least 3 years. SIRTF's three focal plane instruments will permit imaging and spectroscopy over the infrared spectrum from 3 to $200 \mu \mathrm{m}$ with sensitivities of 100 to 10,000 times their predecessors. It will be operated as a facility for the entire scientific community.

\section{EDISON}

EDISON has been proposed to be a long-lived international infrared space observatory possessing a 1.7$\mathrm{m}$ mirror cooled via radiation to approximately $20 \mathrm{~K}$. The observatory will be equipped with imaging and specroscopic instruments operating over the wavelength range 2-100 microns. High sensitivity, high angular resolution, and long life due to lack of cryogens will atlow EDISON to undertake a comprehensive research programme impossible with any other existing or proposed facility.

\section{Submillimeter Intermediate Mission (SMIM)}

SMIM is a proposed submillimeter space telescope with a radiation cooled 2.5-meter diameter primary mirror. The focal-plane instruments include heterodyne receivers that will cover the band from 250-700 microns. Between 100 and 300 microns, a Fabry-Perot spectrometer would provide the spectral coverage. Cooling of the instruments would be provided by a 500-liter cryostat. The mission lifetime is projected to be about 1 year. The orbit attainable using the Delta launch vehicle would be a 24-hour elliptical (if an Atlas II were used, a heliocentric orbit would be preferred).

\section{Pronaos}

Pronaos is a 2-meter diameter balloon-borne telescope for submillimeter astronomy built in France by CNES (Toulouse). The telescope will accommodate two focal-plane instruments: a multiband photometer and a heterodyne spectrometer. The first flight will occur in 1994. 
Far Infrared and Submillimeter Space Telescope (FIRST)

FIRST is a cornerstone mission of the ESA long term science program. It is a large ( 3 meter) diameter passively cooled telescope equipped with a combination of photometer/camera and very high resolution spectrometers for far-infrared and submillimeter wavelengths. FIRST was selected to be the ESA Cornerstone Mission 4, to be launched in 2006.

\section{Stratospheric Observatory for Infrared Astronomy (SOFIA)}

SOFIA will be a 2.5-meter ambient temperature telescope mounted in a specialiy modified Boeing 747 aircraft, which will replace the Kuiper Airborne Observatory around the year 2000. It will have capabilities throughout the spectrum from 0.3 microns to $1.3 \mathrm{~mm}$ wavelength. The planned flight program of 120 8-hour flights per year would support approximately 15 science instruments and 40 principal investigator teams annually. In addition, SOFIA will have a strong educational outreach program. SOFIA will be sponsored by NASA, with possible participation by the German space agency, DARA.

\section{ACKNOWLEDGEMENTS}

The author gratefully acknowledges the assistance of Charles Beichman, Edward H. Dunham, Edward Erickson, Carol Lonsdale, Joseph Mazzarella, and Gary Melnick in the preparation of this review.

\section{REFERENCES}

Adams, F.C., Emerson, J.P. and Fuller, G.A. 1990, Ap.J., 357, 606.

Armus, Heckman, and Miley 1990, Ap.J., 364, 471.

Beckwith, S., Sargent, A.I., Chini, R.S., Güsten, R. 1990, Astron. J., 99, 924.

Beichman, C.A., Boulanger, F., and Moshir, M.M. 1992, Ap. J., 386, 248.

Beichman, C.A, Myers, P.C., Emerson, J.P, Harris, S., Mathieu, R., Benson, P.J., and Jennings, R.E. 1986, Ap.J., 307, 337 .

Blitz, L. and Spergel, D. N. 1991, Ap. J., 379, 631

Boulanger, F., Beichman, C., Désert, F.X., Helou, G., Pérault, and Ryter, C. 1988, Ap. J., 332, 328.

Brown and vanden Bout 1991, A.J., 102, 1956.

Butner, H.M., Evans, N.J. II, Harvey, P.M., and Mundy, L.G. 1990, Ap.J., 364, 164.

Carral, P., Hollenbach, D. J., Lord, S. D., Colgan, S. W. J., Haas, M. R., Rubin, R. H., and Erickson, E. F. 1993, Ap.J., in press.

Cohen, M., Emeison, J.P., and Beichman, C.A. 1989, Ap. J., 339, 455.

Colgan, S. W. J., Haas, M. R., Erickson, E. F., Lord, S. D., and Hollenbach, D. J. 1993, Ap.J., submitted.

Désert, F.X., Boulanger, F., and Puget J-L. 1990, Asiron. \& Astrop., 237, 215.

Djorgovski, G. and Sosin, C. 1989, Ap. J., 341, L13.

Elston et al. 1993, in press.

Erickson, E. F., Haas, M. R., Colgan, S. W. J., Lord, S. D., Burton, M. G. 1988.

Erickson, E. F., Colgan, S. W. J., Simpson, J. P., Rubin, R. H., Morris, M., and Haas, M. R. 1991, ApJ, 370, 69 .

Erickson, E. F., Colgan, S. W. J., Simpson, J. P., Rubin, R. H., and Haas, M. R. 1993, in preparation.

Gauvin, L.S. and Strom, K.M., 1992, Ap. J., 385, 217.

Gehrz, R. D., Woodward, C. E., Greenhouse, M. A., Starrfield, S., Wooden, D. H., Witteborn, F. C., Sandford, S. A., Allamandola, L. J., Bregman, J. D., and Klapisch, M. 1993, Astrophys. J., , in press.

Girard, M., Pajot, F., Caux, E., Lamarre, J.M, Serra, G. 1989, Astr. Astrp., 215, 92.

Goldader et al. 1993, in press.

Golombek, Miley and Neugebauer 1988, A.J., 95, 26.

Gosh, S. K., Iyengar, K. V. K., Rengarajan, T. N., Tandon, S. N., Verma, R. P., and Daniel, R. R. 1990a, ApJ, 353, 564. 
Gosh, S. K., Iyengar, K. V. K., Rengarajan, T. N., Tandon, S. N., Verma, R. P, Daniel, R. R., and Ho, P. T. P. 1990b, ApJ, 347, 338.

Gosh, S. K., Bisht, R. S., Iyengar, K. V. K., Rengarajan, T. N., Tandon, S. N. and Verma, R. P. 1992, ApJ, $391,111$.

Haas, M. R., Colgan, S. W. J., Erickson, E. F., Lord, S. D., Burton, M. G. 1990.

Jackson, J.M., N. Geis, R. Genzel, A.I. Harris, S.C. Madden, A. Poglitsch, G.J. Stacey, and C.H. Townes 1993, ApJ., 402, 173.

Habing ,H.J, Olnon, F.M., Chester, T., Gillett, F.C., Rowan-Robinson, M. and Neugebauer, G. 1985, Astr. Astrp, 152, 11.

Heckman, Armus and Miley 1990, Ap.J.S., 74, 833.

Helou, G. 1986, Ap. J., 311, L33.

Helou, G., Ryter, C., and Soifer, B.T. 1991, Ap. J., 376, 505.

Hildebrand, R.H., Davidson, J.A., Dotson, J., Figer, D.F., Novak, G., and Platt, S.R. 10 Nov.1993, Ap.J., in press.

Hildebrand, L.A., Strom, S.E., Vrba, F. J., Keene, J. 1992, Ap. J., in press.

Hines 1991, Ap.J., 374, L9.

Holenstein, A. P., Schenker, G., Huguenin, D., and Kneubuhl, F. K. 1992, Astron. Astrophys. Suppl. Ser. 96, 115 .

Keene, J. and Masson, C. 1990, Ap.J, 355, 635.

Kenyon, S., Hartmann, L.W., Strom, K.M., Strom, S.E. 1990, Astron. J., 99, 869.

Kenyon et al. 1989.

Kormendy and Sanders 1992, Ap.J., 390, L53.

Lawrence et al. 1993, in press.

Lees, Knapp, Rupen, and Phillips 1990, Ap.J., 379, 177

Lonsdale, Smith and Lonsdale 1993, Ap.J., L9.

Madden, S.C., Geis, N., Genzel, R., Herrmann, F., Jackson, J.M., Poglitsch, A., Stacey, G.J., and Townes, C.H. 1993, Ap.J., 407, 579.

Mazzarella et al. 1991, A.J., 102, 1241.

Mazzarella, Graham, Sanders and Djorgovski 1993, Ap.J., 409, 170.

Morris, M., Davidson, J.A., Werner, W., Dotson, J., Figer, D.F., Hildebrand, R.H., Novak, G., Platt, S.R. 1992, Ap.J., 399, L63.

Mouri and Taniguchi 1992, Ap.J., 386, 68.

Myers, P.C., Fuller, G.A., Mathieu, R. D., Beichman, C.A., Benson, P.J., Schild, R. E., and Emerson, J.P. 1987, Ap.J., 319, 340.

Nakajima et al. 1991, Ap.J., 373, 452.

Natta, A., Palla, F., Butner, H.M., Evans, N.J. II, and Harvey, P.M. 1993, Ap.J., 406, 674.

Natta, A., Palla, F., Butner, H.M., Evans, N.J. II, and Harvey, P.M. 1992, Ap.J., 391, 805.

Poglitsch, A., G.J. Stacey, N. Geis, M. Haggerty, J. Jackson, M. Rumitz, R. Genzel, and C.H. Townes 1991, $A p J$, , 374, L33.

Prusti, T., Whittet, D.C.B. and Wesselius, P.R. 1991, Mon. Not. Roy. Astr. Soc, in press.

Puget, J-L and Léger, A. 1989, Ann. Rev. Astron. \& Astrop., 27, 161.

Rowan-Robinson, M., and Chester, T. 1987, Ap. J., 313, 413.

Rowan-Robinson, M. et al. 1991, Nature, 351, 719.

Rowan-Robinson, M. et al. 1993, MNRAS, in press.

Rucinski, S.M. 1985, Astron. J., 90, 2321.

Salama, F., Allamandola, L. J., Sandford, S. A., Bregman, J. D., Witteborn, F. C., and Cruikshank, D. P. 1993, Icarus, in press.

Salama, F., Allamandola, L. J., Witteborn, F. C., Cruikshank, D. P., Sandford, S. A., and Bregman, J. D. 1990, Icarus, 83, 66 .

Sargent, A. I. and Beckwith, S. 1987, Ap. J., 323, 294.

Sargent, A.I. and Beckwith, S. 1991, Ap.J., 382, L31.

Shibai, H., Okuda, H., Nakagawa, T., Matsuhara, H., Maihara, T., Mizutani, K., Kobayashi, Y.,Hiromoto, N., Nishimura, T., and Low, F. J. 1991, ApJ, 374, 522. 


\title{
3. Optical Astronomy from Space
}

\author{
J.B. Hutchings
}

This section summarises the publications arising from Hipparcos and the visible range results from the Hubble space Telescope, between July 1990 and June 1993. Both telescopes have considerable history and also will continue to produce publications in this areas in the future, so the summary is arbitrarily restricted by the dates covered.

\section{HIPPARCOS}

Ths Hipparcos mission did not achieve its intended orbit at launch, but creative reprogramming of the science resulted in a highly successful mission. Naturally, much of the published literature deals with the orbit and revision of the methods necessary to analyse the data. Some 170 papers are found in the reporting interval. Of about 100 publications which are published in the refereed literature, 33 are found in a special issue of Astronomy and Astrophysics vol 258, May 1992. A summary of the mission was published in ESA SP-1154 (1992). There are also several papers in double stars in IAU Colloquium 134 (PASP conference series), in symposia 7,8 of the Cospar 1990 conference, and IAU symposium 141.

Overall, most of the papers are on data analysis methods, with another significant subset on the orbit and associated problems. There are also many discussions on the catalogues of stars associated with the mission. The main catalogue is the Hipparcos Input Catalogue, which

was published in 7 volumes in March 1992 (Turon et al), and is in itself an excellent reference. Many early paper are on binary and multiple stars. While the final main product of the mission will be some time in the future, progress is very encouraging.

\section{HUBBLE SPACE TELESCOPE}

The Hubble space Telescope has been in operation since mid-1990, with a slow start to science observations due to the spherical aberration of the primary mirror, and initial tracking jitter from solar panel oscillations. The aberration has most adversely affected the visible range science, since the main driver at optical wavelengths is spatial resoiution. Here too much of the literature has been devoted to dealing with the image aberrations, and optical range science has been restricted to objects of relatively high brightness and low dynamic range.

The instruments delivering optical wavelength science are the FOS/RD, WF/PC, and FOC. While the FGS and HSP have been used for optical science, there are no scientific results from them in the literature yet. The innovativ 'snapshot' program, which takes (mostly unguided) pictures from a large catalogue of related targets, to fill in unscheduled time, has yielded significant science.

In the reporting interval some 470 publications are found, which do not involve UV science. Of these, perhaps $1 / 2$ are in refereed journals, of which some $10 \%$ refer to deconvolution methods for the images, and another $10 \%$ to the optical performance of the instruments. There are $22 \%$ extragalactic papers. The remaining topics, in decreasing order of publications, are galactic stars or objects, planetary science, gravitational lenses (snapshot program), globular clusters, and LMC objects.

Extragalactic targets have largely been the central regions of active galaxies and the central light cusps of nearby glaxies. Both focus on trying to understand the nature of galactic nuclei. Generally, in the case of AGN, there is evidence for a bi-conical region illuminated by the central light source, strongly supporting the existing ideas of an opaque torus 
surrounding the central source and broad-line region (e.g. NGC 1068, NGC 4151). Several galaxies have small light cusps that are close to being too small to arise from star clusters. Other unexpected results were crossed dust lanes in the centre of M51; a dust disk in the centre of NGC 4261 double nucleus in M31; young clusters near the cenre of NGC 1275 .

Another category of source is jets, and the braided jets of $3 \mathrm{C} 66 \mathrm{~B}$ and the struture in the $M 87$ and $3 \mathrm{C} 273$ jets are the more celebrated of these. The medium-deep parallel imaging survey has started producing published papers on faint objects in the distant universe. other primary imagi targets have been distant radio galaxies, and Butcher-oemler clusters.

Galactic objects have prominently included the orion nebula, eta carinae, $R$ Aquarii. LMC objects include the R136 field in the centre of 30 Dor, the remnant and light echoes of SN1987a, planetary nebulae. The globular clusters include 47 Tuc and M15, and optical identifications of cluster $\mathrm{X}$-ray sources have been made.

In all these targets, the HST resolution has been significantly better. than ground-based, and major new morphological information has been obtained. Solar system work has mainly been devoted to the bright planets, with detailed imaging of Mars, Jupiter, and Saturn. The great white strom on Saturn in oct/Nov 1990 was the subject of an extended special program.

There are many new unpublished results, and the HST publications are ramping up rapidly. It remains to be seen what difference the proposed optical fixes will produce in future work: it should alter the balance of HST science significantly.

Summaries of HST science, including UV as well as optical, are published in PASP (Kinney and Maran 1991, Maran and Kinney 1993) and also the conference proceedings from the Space Telescope Institute (Kinney and Blades 1991) and Chia Laguna, Cagliari (Benvenuti and Schreier 1992).

Selected reference list

Hipparcos references

Bec-Borsenberger, A.

Ephemerides of the 48 Hipparcos minor planets for the year 1993

Astronomy \& Astrophysics Suppl 1 April 1993; 98(1); P. 77-125; 6 REFS

Canuto, E.; Donati, F.

The on-ground attitude reconstitution of the Hipparcos satellite

International Journal of Modelling and Simulation $1992 ; 12(4) ; P .125-30$

Seille, J.; Mazeau, P.; Garres, C. ; Borde, J.

Environmental prediction and radiation effects of protons into solar generators: application to HIPPARCOS revised mission

RADECS 91: First European Conference on Radiation and its Effects on Devices and Systems (Cat. No.91TH0400-2)

Publ: IEEE; New York, NY, USA 1992; P. 307-11; 593; 0 REFS

Pannunzio, R.; Morbidelli, R.; Massone, G.; Lattanzi, M.G.; Sarasso, M.; Chiumiento, G.

Precise equatorial coordinates of double and multiple systems: an astronomical support to the Hipparcos mission

Astronomy \& Astrophysics Suppl Nov. 1992; 96(1); P. 143-55; 12 REFS

Brosche, P.; Denis-Karafistan, A.; Denis, C.

Determination of the optical or physical nature of wide visual binaries

by means of two-dimensional conditional probabilities

Astronomische Nachrichten $1992 ; 313(6) ;$ P. 341-7; 7 REFS

Fehrenbach, Ch.; Burnage, R.; Figuiere, J.

Radial velocities. VI. Ground based measurements for Hipparcos

Astronomy \& Astrophysics Suppl Nov. 1992; 95(3); P. 541-79; 12 REFS 
ESA's Report to the 29th COSPAR Meeting (ESA SP-1154)

Publ: ESA; Noordwijk, Netherlands 1992; 188

Turon, c. et al

The Hipparcos Input Catalogue

Bulletin d'Information du Centre de Donnees Astronomiques de Strasbourg Juiy 1992; no.41; P. 9-20; 31 REFS

Duflot, M.; Fehrenbach, C.; Mannone, C.; Burnage, R.; Genty, V. Radial velocities. V. Ground based measurements for Hipparcos

Astronomy \& Astrophysics Suppl sept. 1992; 94(3); P. 479-517; 10 REFS

Makarov, V.V.

Periodical errors in the Hipparcos star abscissae, caused by oscillations of the basic angle

Pis'ma $V$ Astronomicheskie Zhurnal 1992; 18(7); P. 630-7; 3 REFS

Vondrak, J.; Feissel, M.; Essaifi, N.

Expected accuracy of the 1900-90 Earth orientation parameters in the Hipparcos reference frame

Astronomy and Astrophysics Aug. 1992; 262(1); P. 329-40; 18 REFS

Turon, c. et al

Hipparcos Input Catalogue

March $1992 ; 7$ vol. $(2377+399+437)$

Egret, D.; Didelon, P.; Mclean, B.J.; Russell, J.L.; Turon, C.

The Tycho Input Catalogue. Cross-matching the Guide star Catalog with the Hipparcos INCA Data Base

Astronomy and Astrophysics May 1992; 258(1); P. 217-22; 20 REFS

Scales, D.R.et al

Tycho photometry calibration and first results

Astronomy and Astrophysics May 1992; 258(1); P. 211-16; 10 REFS

Wicenec, A.; Bassgen, G.

Tycho background determination and monitoring

Astronomy and Astrophysics May 1992; 258(1); P. 206-10; 10 REFS

Hog, E. et al

Tycho astrometry calibration

Astronomy and Astrophysics May 1992; 258(1); P. 201-5; 11 REFS

Halbwachs, J.L.; Hog, E.; Bastian, U.; Hansen, P.C.; Schwekendiek, P. Tycho star recognition

Astronomy and Astrophysics May 1992; 258(1); P. 193-200; 8 REFS

Bassgen, G. et al

Tycho transit detection

Astronomy and Astrophysics May 1992; 258(1); P. 186-92; 11 REFS

Hog, E. et al

Tycho data analysis. Overview of the adopted reduction software and first results

Astronomy and Astrophysics May 1992; 258(1); P. 177-85; 12 REFS

Pannunzio, $R$. et al

The treatment of Hipparcos observations of some peculiar double stars: anomalous cases

Astronomy and Astrophysics May 1992; 258(1); P. 173-6; 10 REFS 
Mignard, F. et al

Hipparcos double star recognition and processing within the FAST consortium

Astronomy and Astrophysics May 1992; 258(1); P. 165-72; 10 REFS

Soderhjelm, S.; Evans, D.W.; van Leeuwen, F.; Lindegren, L.

Detection and measurement of double stars with the Hipparcos satellite: NDAC reductions

Astronomy and Astrophysics May 1992; 258(1); 2. 157-64; 13 REFS

Evans, D.W.; van Leeuwen, F.; Penston, M.J.; Ramamani, N.; Hog, E. Hipparcos photometry: NDAC reductions

Astronomy and Astrophysics May 1992; $258(1) ;$ P. 149-56; 10 REFS

Mignard, F.; Froeschle, M.; Falin, J.L.

Hipparcos photometry: FAST main mission reduction

Astronomy and Astrophysics May 1992; 258(1); P. 142-8; 8 REFS

Lindegren, $\mathrm{L}$. et al

Positions and parallaxes from the Hipparcos satellite. A first attempt at a global astrometric solution

Astronomy and Astrophysics May 1992; 258(1); P. 134-41; 11 REFS

Turon, C.; Arenou, F.; Evans, D.W.; van Leeuwen, F.

Comparison of the first results from the Hipparcos star mappers with the Hipparcos Input Catalogue

Astronomy and Astrophysics May 1992; 258(1); P. 125-33; 23 REFS

van Leeuwen, F.; Evans, D.W.; I.indegren, L.; Penston, M.J.; Ramamani, N. Early improvements to the Hipparcos Input Catalogue through the accumulation of data from the satelite including the NDAC attitude reconstruction description

Astronomy and Astrophysics May 1992; 258(1); P. 119-24; 17 REFS

Crifo, F.; Gomez, A.; Arenou, F.; Morin, D.; Schrijver, H. Reliability of the Hipparcos. Input Catalogue tested by the 'First look' Astronomy and Astrophysics May 1992; 258(1); P. 116-18; 10 REFS

Lestrade, J.-F.; Phillips, R.B.; Preston, R.A.; Gabuzda, D.C. High-precision VLBI astrometry of the radio-emitting star sigma CrB-a step in linking the Hipparcos and extragalactic reference frames Astronomy and Astrophysics May 1992; 258(1); P. 112-15; 17 REFS

Arenou, F.; Grenon, M.; Gomez, A.

A tridimensional model of the Galactic interstellar extinction

Astronomy and Astrophysics May 1992; 258(1); P. 104-11; 17 REFS

Mennessier, M.O.; Barthes, D.; Boughaleb, H.; Figueras, F.; Mattei, J.A.. Computation of ephemerides for long-period variable stars for the Hipparcos mission

Astronomy and Astrophysics May 1992; 258(1); P. 99-103; 24 REFS

Bec-Borsenberger, $A$.

Solar system objects observed by Hipparcos

Astronomy and Astrophysics May 1992; 258(1); P. 94-8; 15 REFS

Grenon, M.; Mermilliod, M.; Mermilliod, J.C.

The Hipparcos Input Catalogue. III. Photometry

Astronomy and Astrophysics May 1992; 258(1); P. 88-93; 8 REFS

Jahreiss, H. et al

The Hipparcos Input catalogue. II. Astrometric data

Astronomy and Astrophysics May 1992; 258(1); P. 82-7; 28 REFS 
Turon, C. et al

The Hipparcos Input Catalogue. I. Star selection

Astronomy and Astrophysics May 1992; 258(1); P. 74-81; 82 REFS

Schrijver, $\mathrm{H}$.

Comparison of Hipparcos results obtained on different dates on the same great circle

Astronomy and Astrophysics May 1992; 258(1); P. 70-3; 12 REFS

van der Marel, H.; Petersen, C.

Hipparcos great-circle reduction. Theory, results and intercomparisons

Astronomy and Astrophysics May 1992; 258(1); P. 60-9; 15 REFS

van Leeuwen, F.; Penston, M.J.; Perryman, M.A.C.; Evans, D.W.; Ramamani, N. Modelling the torques affecting the Hipparcos satellite

Astronomy and Astrophysics May 1992; 258(1); P. 53-9; 6 REFS

Bernacca, P.L. et al

Early scientific results from Hipparcos and future expectations

ESA Bulletin Feb. 1992; no.69; P. 51-7; O REFS

Hog, E. ; Kovalevsky, J.; Lindegren, L.

Hipparcos data reduction-construction of the Hipparcos Star Catalogue

ESA Bulletin Feb. 1992; no.69; P. 43-50; 0 REFS

Turon, $c$.

The Hipparcos observing programme: preparation of the Input catalogue

ESA Bulletin Feb. 1992; no.69; P. 36-42; 0 REFS

Ratier, G.; van Katwijk, K.; Fade, G.; Perryman, M.A.C.

The Hipparcos payload's in-orbit performance

ESA Bulletin Feb. 1992; no.69; P. 27-32; 0 REFS

Heger, D.; McDonald, A.; Schutz, A.; Ojangurem, O.; Sollazzo, C.

ESOC's role in routine Hipparcos operations

ESA Bulletin Feb. 1992; no.69; P. 16-25; 0 REFS

Van der Ha, $J$.

Implementation of the revised Hipparcos mission at ESOC

ESA Bulletin Feb. 1992; no.69; P. 9-15; 0 REFS

Donati, F.; Sechi, G.

Method of comparison between determinations of the Hipparcos attitude

Astronomy and Astrophysics May 1992; 258(1); P. 46-52; 5 REFS

Donati, F.

Attitude determination in the Hipparcos revised mission

Astronomy and Astrophysics May 1992; 258(1); P. 41-5; 6 REFS

Lindegren, L.; Le Poole, R.S.; Perryman, M.A.C.; Petersen, C.

Geometrical stability and evolution of the Hipparcos telescope

Astronomy and Astrophysics May 1992; 258(1); P. 35-40; 8 REFS

Schrijver, H.; van der Marel, H.

Geometrical calibration and assessment of the stability of the Hipparcos payload

Astronomy and Astrophysics May 1992; 258(1); P. 31-4; 8 REFS

Iindegren, $L$. et al

The NDAC Hipparcos data analysis consortium. Overview of the reduction 


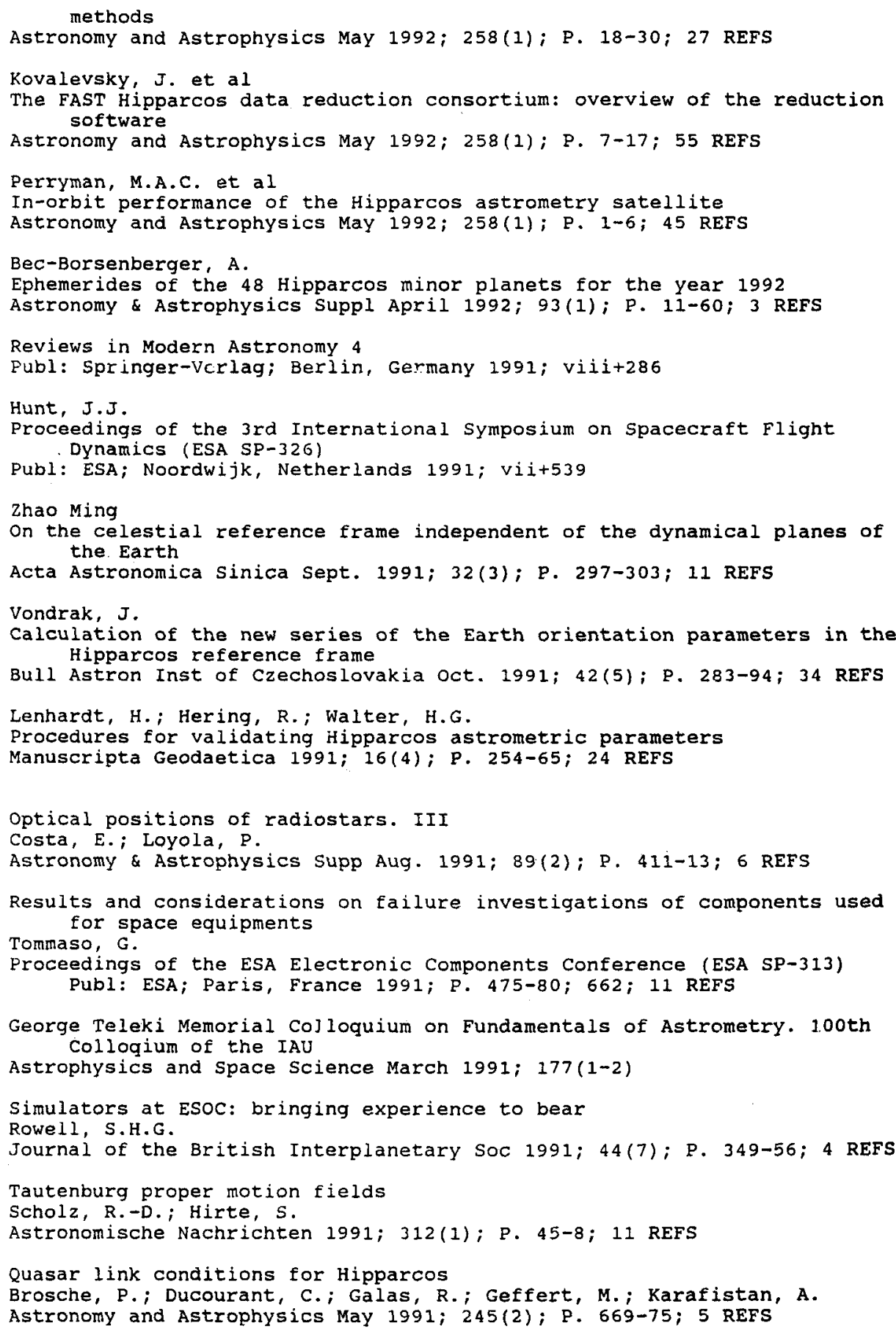


Infrared and Radio Astronomy and Astrometry. Symposia 7,8 and the Topical Meeting of COSPAR Interdisciplinary Scientific Commission $E$ (Meeting 5) of the COSPAR 28 th Plenary Meeting Advances in Space Research 1991; 11(2)

Relative positions and proper motions of components of 32 triple stars from the Hipparcos Input Catalog Anosova, Zh.P.; Orlov, V.V.; Todua, M.M. Kinematika i Fizika Nebesnykh Tel 1990; 6(2); P. 8-12; 3 REFS

UBVRI photometry of G, K, M Hipparcos stars. II

Jordi, C.; Figueras, F.; Paredes, J.M.; Rossello, G. ; Torra, J. Astronomy \& Astrophysics Suppl Feb. 1991; 87(2); P. 229-31; 8 REFS

A catalogue of QSOs and link stars for the HST link of the Hinparcos reference frame

White, G.L.et al

Proceedings of the Astr Soc Australia 1990; 8(4); P. 368-71; 24 REFS

Speckle interferometry of Hipparcos link stars. III

White, G.L. et al

Monthly Notices Royal Astrl Soc 1 Feb. 1991; 248(3); P. 411-13; 10 REFS

Results of the HIPPARCOS in-orbit payload calibration

Davies, P.E.; McDonald, A.J.C.

Journal of the British Interplanetary Soc 1991; 44 (1); P. 37-48; 4 REFS

The Hipparcos mission-on the road to recovery

Hassan, H.; Clausen, K.; Perryman, M.A.C.; van der Ha, J.; Heger, D. ESA Bulletin Nov. 1990; no.64; P. 59-66; 0 REFS

Ephemerides of the 48 Hipparcos minor planets for the year 1991

Bec-Borsenberger, A.

Astronomy \& Astrophysics Suppl Dec. 1990;86(2); P. 299-349;3 REFS

On the problem of the HIPPARCOS reference frame

Lattanzi, M.G.; Bucciarelli, B.; Bernacca, P.L.

Astrophysical Journal Suppl July 1990; 73(3); P. 481-8; 33 REFS

\section{HST References}

O'Dell, C.R.; Wen, Z.; Hu, X.

Discovery of new objects in the orion Nebula on HST images: shocks, compact sources, and protoplanetary disks

Astrophysical Journal 1993; 410(2, pt.1); P. 696-700+7 plates; 35 REFS

Schade, D.J.; Elson, R.A.W.

What do you gain from deconvolution? observing faint galaxies with the Hubble Space Telescope wide field camera

Astronomical Journal April 1993; 105(4); P. 1581-6; 17 REFS

Hollis, J.M.; Michalitsianos, A.G.

Evidence for precession of the $R$ Aquarii jet

Astrophysical Journal 1 July 1993;411(1, pt.1); P. 235-8+1 plate; 23 REFS

Klaus, R.; Burkhardt, H.

Regularized multiframe restoration of Hubble space and ground-based telescope images with parallel implementation

Proceedings of the SPIE - The International society for optical Engineering 1993; 1771; P. 124-34; 23 REFS 
Roddier, C.; Roddier, F.

Combined approach to the Hubble space Telescope wave-front distortion analysis

Applied Optics 1 June 1993; 32(16); P. 2992-3008; 17 REFS

Filippenko, A.; Ho, L.C.; Sargent, W.L.W.

HST observations of NGC 4395, the least luminous seyfert 1 nucleus: evidence against the starburst hypothesis for broad-lined active galactic nuclei

Astrophysical Journal, Letters $1993 ; 410(2$, pt.2); P. L75-8; 31 REFS

De Marchi, G.; Paresce, F.; Ferraro, F.R.

The structure of the core of 47 Tucanae from Hubble space Telescope observations

Astrophysical Journal Suppl April 1993; 85(2); P. 293-314; 32 REFS

Barbieri, c. et al

Compact subarcsec structures of the double nucleus of NGC 6240 revealed with HST

Astronomy and Astrophysics June 1993; 273(1); P. 1-5; 19 REFS

Snyder, D.L.; Hammoud, A.M.

Image recovery from data acquired with a charge-coupled-device camera

Journal of the Optical Soc America A May 1993; 10(5); P. 1014-23; 31 REFS

Maoz, D. et al

The Hubble Space Telescope Snapshot survey. IV. A summary of the search for gravitationally lensed quasars

Astrophysical Journal 20 May 1993; 409(1, pt.1); P. 28-41; 27 REFS

Bendinelli, 0 . et al

The first detection of a collapsed core globular cluster in M31

Astrophysical Journal, Letters 1993; 409(1, pt.2); P. L17-19+1 plate

Harris, H.C.; Hunter, D.A.; Baum, W.A.; Jones, J.H.

Photometric calibration of the HST Wide-Field/Planetary Camera. II. Ground-based observations of calibration fields

Astronomical Journal March 1993; 205(3); P. 1196-205, 1239-42; 7 REFS

Caldwell, J.; Xin-Min Hua; Turgeon, B.; Westphal, J.A.; Barnet, C.D.

The drift of Saturn's north polar spot observed by the Hubble Space Telescope

Science 16 April 1993; 260(5106); P. 326-9; 11 REFS

Jackson, N.; Sparks, W.B.; Miley, G.K.; Macchetto, F.

The radio and optical structure of $3 \mathrm{C} 66 \mathrm{~B}$

Astronomy and Astrophysics March 1993; 269(1-2); P. 128-34; 17 REFS

Kroger, H.W.; Schmidt, G.K.; Pailer, N.

Faint object Camera: european contribution to the Hubble space Telescope Acta Astronautica Nov. 1992; 26(11); P. 827-34; 0 REFS

Benedict, G.F. et al

Astrometric performance characteristics of the Hubble space Telescope Fine Guidance Sensors

Publications of the Astron Soc Pac Oct. 1992; 104(680); P. 958-75; 12 REFS

Barnet, C.D.; Westphal, J.A.; Beebe, R.F.; Huber, L.F.

Hubble space Telescope observations of the 1990 equatorial disturbance on Saturn: zonal winds and central Meridian albedos

Icarus Dec. 1992; 100(2); P. 499-511; 24 REFS

Westphal, J.A. et al

Hubble Space Telescope observations of the 1990 equatorial disturbance 
on Saturn: images, albedos, and limb darkening Icarus Dec. 1992; $100(2) ;$ P. 485-98; 30 REFS

Calzetti, D.; De Marchi, G.; Paresce, F.; Shara, M. The center of gravity and density profile of 47 Tucanae Astrophysical Journal, Letters 1993; 402(1, pt.2); P. L1-4+1 plate

Schmidt, G.D.; Elston, R.; Lupie, O.L.

The Hubble Space Telescope northern-hemisphere grid of stellar polarimetric standards

Astronomical Journal Oct. 1992; 104(4); P. 1563-7; 9 REFS

Guhathakurta, P.; Yanny, B.; Schneider, D.P. ; Bahcall, J.N.

Globular cluster photometry with the Hubble Space Telescope. I. Description of the method and analysis of the core of 47 Tuc Astronomical Journal Nov. 1992; 104(5); P. 1790-817; 57 REFS

Campiell, B. et al

Hubble Space Telescope Planetary Camera images of R136

Astronomical Journal Nov. $1992 ; 104(5) ;$ P. 1721-42+4 plates; 43 REFS

Maoz, D, et al

The Hubble Space Telescope Snapshot Survey. III. Further observations in search of gravitationaliy lensed quasars

Astrophysical Journal 1 Jan. 1993; 402(1, pt.1); P. 69-75; 29 REFS

Miley, G.K.; Chambers, K.C.; van Breugel, W.J.M.; Macchetto, F.

Hubble Space Telescope imaging of distant galaxies: $4 \mathrm{C} 41.47$ at $z=3.8$ Astrophysical Journal, Letters 1992; 401(2, pt.2); P. L69-73+2 plates

Sandage, A.; Saha, A.; Tammann, G.A.; Panagia, N.; Macchetto, D.

The Cepheid distance to IC 4182 ; calibration of $M / s u b V /(\max )$ for $S N$ Ia $1937 \mathrm{C}$ and the value of $\mathrm{H} / \mathrm{sub}$ phi /

Astrophysical Journal, Letters 1992; 401(1, pt.2); P. L7-10+1 plate

Lucks, $M$.

Detecting opportunities for parallel observations on the Hubble Space Telescope

Telematics and Informatics Summer-Fall 1992; 9(3-4); P. 331-47; 11 REFS

Korista, K.T. et al

Hubble Space Telescope Faint object Spectrograph and ground-based. observations of the broad absorption line quasar 0226-1024

Astrophysical Journal 20 Dec. 1992; 401(2, pt.1); P. 529-42; 43 REFS

ESA's Report to the 29th COSPAR Meeting (ESA SP-1154)

Publ: ESA; Noordwijk, Netherlands 1992; 188

Dols, V.; Gerard, J.C.; Paresce, F.; Prange, R.; Vidal-Madjar, A.

Ultraviolet imaging of the Jovian aurora with the Hubble Space Telescope Geophysical Research Letters 23 Sept. 1992; 19(18); P. 1803-6; 17 REFS

Bahcall, J.N.; Hartig, G.F.; Jannuzi, B.T.; Maoz, D.; Schneider, D.P. Faint object spectrograph observations of the large-redshift gravitational lens candidate $1208+1011$

Astrophysical Journal, Letters 1992; 400(2, pt.2); P. L51-4; 23 REFS

Windhorst, R.; Mathis, D.F.; Keel, W.C.

Deep Hubble space Telescope imaging of a compact radio galaxy at $z=2.390$ Astrophysical Journal, Letters $1992 ; 400(1$, pt. 2$) ;$ p. L1-4+3 plates

Thomson, R.C.; Schade, D.J.; Elson, R.A.W.; Mackay, C.D.; Wilkins, T.N. Deconvolution and photometry of Faint object Camera images with point 
Burgarella, D.; Paresce, F.

The structure of the inner arcsecond of $R$ Aquarii observed with the Hubble space Telescope

Astrophysical Journal, Letters $1992 ; 389(1$, pt.2); P. L29-32+1 plate

Bahcal1, J.N. et al

The Snapshot Survey: a search for gravitationally lensed quasars with the Hubble Space Telescope

Astrophysical Journal 1 March $1992 ; 387(1$, pt. 1) ; P. 56-68; 37 REFS

Lauer, T.R. et al

Planetary Camera observations of the M87 stellar cusp

Astronomical Journal March 1992; $1: 3(3) ; \mathrm{P} .703-10,1025-6 ; 33$ REFS

Holtzman, J.A. et al

Planetary Camera observatiors of NGC 1275: discovery of a central population of compact massive blue star clusters

Astronomical Journal March 1992; 103(3); P. 691-702, 1020-3; 53 REFS

Kinney, A.L.; Maran, S.P.

The first year of observations with the Hubble Space Telescope

Publications Astron Soc PaC Dec. 1991; 103(670); P. 1237-49; 78 REFS

Franz, O.G. et al

Binary star observations with the rubble space Telescope fine guidance sensors. II. Bright Hyades

Astronomical Journal Jan. 1992; 103(1); P. 190-6; 10 REFS

Maoz, D. et al

A gravitational lens candidate discovered with the Hubble Space Telescope

Astrophysical Journal, Letters $1992 ; 386(1$, pt.2); P. L1-3; 19 REFS

Hollis, J.M.; Dorband, J.E.; Yusef-Zadeh, F.

Comparing restored HST and VLA imagery of $R$ Aquarii

Astrophysical Journal 1992; $386(1$, pt.1); P. 293-8+4 plates; 22 REFS

Jakobsen, P.; Greenfield, P.; Jedrzejewski, R.

The cramer-Rao lower bound and steilar photometry with aberrated HST images

Astronomy and Astrophysics Jan. 19פ2; $253(1) ;$ P. 329-32; 9 REFS

Magain, P.; Surdej, J.; Vanterriest, C.; Pirenne, B.; Hutsemekers, D. Q 1208+1011: the most distant imaged quasar, or a binary?

Astronomy and Astrophysics Jan. 1992; 253(2); P. L13-16; 29 REFS

King, I.R. et al

The current ability of HST to reveal morphological structure in medium-redshift galaxies

Astronomical Journal Oct. 1991; $102(4) ;$ P. 1553-68; 31 REFS

Paltoglou, G.; Bell, R.A.

Theoretical colours and isochrones for some Hubble space Telescope colour systems. II

Monthly Notices Royal Astron Soc 1 Jec. 1991; 253(3); P. 449-73; 50 REFS

Weigelt, $G$. et al

First results from the Faint objec: Camera: high-resolution observations of the central object $R 136$ ir. the 30 Doradus nebula

Astrophysical Journal, Letters 1991; 378(1, pt.2); P. L21-3+3 plates

Hubble Space Telescope imaging of eta Carinae 
sources

Monthly Notices Royal Astron Soc 1 Nov. 1992; 259(1); P. 104-14; 18 REFS

Rix, H.-W.; Schneider, D.P.; Bahcall, J.N.

Hubble Space Telescope Wide Field Camera imaging of the gravitational lens $2237+0305$

Astronomical Journal Sept. 1992; 104(3); P. 959-67, 1268-9; 32 REFS

Bahcall, J.N.; Maoz, D.; Schneider, D.P.; Yanny, B.; Doxsey, R.

Hubble space Telescope imaging of the large-redshift gravitational lens candidate $1208+1011$

Astrophysical Journal, Letters $1992 ; 392(1$, pt.2); P. L1-4+2 plates

Lauer, T.R. et al

Planetary Camera observations of the central parsec of M32

Astronomical Journal Aug. 1992; 104(2); P. 552-62; 32 REFS

Caldwell, J.; Turgeon, B.; Hua, X.-M.

Hubble space Telescope imaging of the north polar aurora on Jupiter

Science 11 Sept. 1992; 257(5076); P. 1512-15; 11 REFS

Hershey, J.L.

A deconvolution technique for Hubble space Telescope FGs fringe analysis

Publications of the Astron SoC PaC JulY 1992; 104(677); P. 592-6; 4 REFS

Blades, J.C. et al

Imaging of four planetary nebulae in the Magellanic clouds using the Hubble Space Telescope Faint Object Camera

Astrophysical Journal, Letters $1992 ; 398(1$, pt. 2$) ;$ P. L41-4; 23 REFS

Cheng, F.H. et al

The Hubble space Telescope Observations of X-ray nova muscae 1991 and its spectral evolution

Astrophysical Journal 1 Oct. 1992; 397(2, pt.1); P. 664-73; 32 REFS

Elson, R.A.W.; Schade, D.J.; Thomson, R.C.; Mackay, C.D.

The massive stars in the R136 region of 30 Doradus

Monthly Notices Royal Astron Soc I Sept. 1992; 258(1); P. 103-6+2 plates

Arp, H.; Crane, P.

Testing the gravitational lens hypothesis in $62237+0305$

Physics Letters A 10 Aug. 1992; 168(1); P. 6-12; 13 REFS

Workshop on the Martian Surface and Atmosphere Through Time (ITI/TR 92-02)

Publ: Lunar \& Planetary Inst; Houston, TX, USA 1992; xvii+182

Weaver, H.A. et al

Inner coma imaging of Comet Levy (1990c) with the Hubble space Telescope Icarus May 1992; $97(1) ;$ P. 85-98; 26 REFS

Caldwell, J. et al

Titan: evidence for seasonal change-a comparison of Hubble space Telescope and Voyager images

Icarus May 1992; $97(1) ;$ P. 1-9; 17 REFS

Maoz, D. et al

Gravitational lensing of quasars as seen by the Hubble Space Telescope Snapshot Survey

Astrophysical Journal 1992; $394(1$, pt. 1$) ;$ P. 51-60 +1 plate; 32 REFS

Crane, P.; Stiavelli, M.

Resolution of the southern radio lobe of $3 \mathrm{C} 33$ at $660 \mathrm{~nm}$

Monthly Notices Royal Astron Soc 1 July 2992; 257(1); P. 17p-20; 15 REFS 
The post collapse core of M15 imaged with the HST Planetary Camera Lauer, T.R. et al

Astrophysical Journal, Letters $1991 ; 369$ (2, pt); P. L45-9 + 2 plates

The core of the nearby SO galaxy NGC 7457 imaged with the HST Planetary Camera

Laver, T.R. et al

Astrophysical Journal, Letters $1991 ; 369$;2, pt.2; P. L41-4+1 plate

Stellar photometry with the Hubble Space Telescope Wide-Field/Planetary Camera: a progress teport

Holtzman, J.A. et al

Astrophysical Journal, Letters 1991; 369(2, pt.2); P. L35-40+2 plates

NGC 1068: resolution of nuclear structure in the optical continuum Lynds, R. et al

Astrophysical Journal, Letters 1991; 369(2, pt.2); P. I31-4+3 plates

HST i.maging of the inner 3 arcseconds of NGC 1068 in the light of (O III) Lambda 5007

Evans, I.N. et al

Astrophysical Journal, Letters 1991; 369(2, pt.2); P. L27-30+2 plates

Tests of the photometric accuracy of image restoration using the maximum entropy algorithm

Cohen, J.G.

Astronomical Journal Feb. 1991; 101(2); P. 734-7; 13 REFS

Photometric calibration of the HST Wide-Field/Planetary Camera. I. Ground-based observations of standard stars

Harris, H.C.; Baum, W.A.; Hunter, D.A.; Kreidl, T.J.

Astronomical Journal Feb. 1991; 101(2); P. 677-94; 17 REFS

Saturn's Great White Spot spectacular

o'Meara, S.J.

Sky and Telescope Feb. 1991; 81(2); P. 144-7; 0 REFS

A catalogue of QSOS and link stars for the HST link of the Hipparcos reference frame

White, G.L. et al

Proceedings Astron Soc Australia 1990; 8(4); P. 368-71; 24 RE:S

Ground-based $\mathrm{CCD}$ observations of two $\mathrm{OB}$ associations in M31 obtained through replicas of two wide Field/Planetary camera filters

Hunter, D.A.; Winkelman, S.

Publications Astron SoC PaC Aug. 1990; 102(654); P. 854-64; 37 REFS

A uvby-like photometric system for the WF/PC of the HST

Kiselman, D.; Oja, T.; Gustafsson, B.

Astronomy and Astrophysics Nov. 1990;238(1-2); P. 265-9; 11 REFS

Stellar photometry with the Wide Field/ Planetary Camera of the Hubble Space Telescope

Holtzman, J.A.

Publications Astron Soc Pac July 1990; 102(653); P. 806-34; 10 REFS

Maran, S.P.; Kinney, A.L.

The second year of observations with the Hubble Space Telescope

Publications Astron Soc Pac May 1993; 
Hester J.J. et al

Astronomical Journal Aug. 1991; 102 (2); P. 654-7; 26 REFS

Binary star observations with the Hubble Space Telescope Fine Guidance Sensors.I. ADS 11300

Franz, O.G. et al

Astrophysical Journal, Letters 1991; 377 (1, pt.2); P. L17-20: 5 REFS

Faint Object Spectrograph observation of the low luminosity Seyfert galaxy NGC 1566

Kriss, G.A.; Harting, G.F.; Armus, L.; Blair, W.P.; Caganoff, S.: Dressel, L.

Astrophysical Journal, Letters 1991; 377(1, pt.2); P. L13-16; 36 REFS

FOS spectroscopy of resolved structure in the nucleus of NGC 1068 Caganoff, S. et al

Astrophysical Journal, Letters 1991; 377(1, pt.2); P. L9-12; 15 REFS

First. results form the Faint object Camera: high-resolution imaging of the Pluto-Charon system

Albrecht, $R$. et al

Astrophysical Journal, Letters 1991; 374(2, pt.2); P. L65-7+1 Plate

HST observations of $3 \mathrm{C} 66 \mathrm{~B}$ : a double-stranded optical jet

Macchetto, F. et al

Astrophysical Journal, Letters 1991; 373(2, pt.2); P. L55-8+2 plates

Blue stragglers in the core of the globular cluster 47 Tucanae

Paresce, F. et al

Nature 25 July 1991; 352(6333); P. 297-301; 26 REFS

Ionization fronts and shocked flows: the structure of the orion Nebula at $0.1 "$

Hester, J.J. et al

Astrcphysical Journal, Letters 1991; 369(2, pe.2); P. L75-8+4 plates

Faint object Camera observations of a globular cluster nova field

Margon, B.; Anderson, S.F.; Downes, R.A.; Bohlin, R.C.; Jakobsen, P.

Astrophysical Journal, Letters $1991 ; 369(2, p t .2) ;$ P. L71-4t1 plate

First results from the Faint object Camera: imaging the core of $\mathbf{R}$ Aquarii

Paresce, F. et al

Astrophysical Journal, Letters 1991; 369(2, pt.2); P. L67-70+1 plate

First results from the Faint object Camera: SN 1987A

Jakobsen, P. et al

Astrophysical Journal, Letters 1991; 369(2, pt.2); P. L63-6+1 plate

First results from the Faint object Camera: images of the gravitational lens system $\mathrm{G} 2237+0305$

Crane, P. et al

Astrophysical Journal, Letters 1991; 369(2, pt.2); P. L59-61+1 plate

First results from the Faint object Camera: observations of PKS $0521-36$

Macchetto, F. et al

Astrophysical Journal, Letters $1991 ; 369(2$, pt.2); P. L55-7+2 plates

Hubble space Telescope Wide-Field/Planetary Camera images of Saturn Westphal, J.A. et al

Astrophysical Journal, Letters 1991; 369(2, pt.2); P. L51-3+3 plates 


\section{X-Ray Astronomy}

Joachim E. Trümper

\section{INTRODUCTION}

The past triennium (July 1990 - June 1993) has seen enormous progress in X-ray astronomy. The following satellite/instruments were operating in orbit

-Ginga (Japan/UK, 1987-1991):

$\mathrm{X}$-ray spectra and time variability studies $(2-30 \mathrm{keV})$.

-Mir-Kvant International Observatory (Russia, Germany, The Netherlands, UK, 1987 - ):

broad band $X$-ray spectra and ) time variability $(2-1000 \mathrm{keV}, \mathrm{X}$-ray imaging $(2-20 \mathrm{keV})$.

-ROSAT (Germany, US,UK, 1990 -):

soft X-ray all-sky survey $(0.1-2.5 \mathrm{keV})-4000$ pointed observations; $X$-ray spectra, imaging time variability. deep surveys.

-Granat (Russia, Francc. Denmark)

$\mathrm{X}$-ray imaging , time variability $(2-20 \mathrm{keV})$.

-BBR XT (US 1990):

$\mathrm{X}$-ray spectroscopy.

-ASCA (Japan, US, 1993-):

broad band $\mathrm{X}$-ray imaging $(0.5-10 \mathrm{keV})$ spectrocopy.

\section{X-RAY EMISSION FROM NORMAL STARS AND WHITE DWARFS}

ROSAT: Discovery of X-rays from the bright and dark sides of the Moon; discovery of metal absorption in white dwarfs; discovery of self-absorption in hot star winds; detection of shocks in hot star winds; detection of $X$-ray emission from hybrid stars; detection of the latest X-ray emitting star (LHS3003); discovery of longduration flares in dMe stars; detection of flares in hybrid stars; detection of flares in low-activity giants; detection of flares in F stars; detection of superflares on RS CVn systems; discovery of microflares in dMe stars; determination of coronal structure on a G-type dwarf; detection of eclipses in Algol and YY Gem; detection of an eclipse of the high-temperature component in AR Lac; detection of the strongest flare on a classical T Tauri star: ubiquitous distribution of weak line T Tauri stars over star forming regions (J. Schmitt, 1992, 1994).

Ginga: Detection of flare thermal emission up $18 \mathrm{keV}$ in Il Peg (Doyle et al., 1991).

\section{X-RAY EMISSION FROM SINGLE NEUTRON STARS}

ROSAT: Discovery of X-rays from the radio pulsars PSR 0950-08, PSR 0823+26, PSR 1823-13, PSR $1951+32$ (associated with CTB80)

Discovery of pulsed X-rays from the radio pulsars PSR 1929+10, PSR 0833-45 (Vela pulsar), PSR 1706-44, PSR 1055-52, PSR 0656+15

Discovery of X-rays from the millisecond pulsar PSR $1957+20$

Discovery of pulsed X-rays from the millisecond pulsar PSRJ 0437-4715 (Becker and Trümper, 1993) 
Discovery of pulsed X-rays from the Gamma source Geminga - first detection of a single neutron star outside the radio band (Halpern and Holt. 1992)

\section{CATACLYSMIC VARIABLES}

Due to the ROSAT survey the number of X-ray detected CVs has doubled. The fraction of magnetic CVs is $\leq$ $80 \%$, that of old novae and nova-like variables $\geq 20 \%$. Two AMHer systems were found in the period gap ( 125.5 and $140 \mathrm{~min}$ ). X-ray emission from an optically thick boundary layer could only be observed in an outburst of SS Cyg, but not in an outburst of 9 other sources (Bunk and Verbunt, 1994).

\section{X-RAY BINARIES}

Granat: discovery and localization of three galactic sources with emission features at approximately $511 \mathrm{keV}$ (IE1740-2942, X-Ray Nova Muscae and Crab Nebula); three X-ray Novae were discovered (Nova Muscae, Nova Oph 1993, Nova Vela 1993); discovery of QPO from four black hole candidates: GX 339-4, X-Ray Nova Muscae, X-ray Nova Persei and Cyg X-1; mapping of the galactic center region in the spectral band 3$800 \mathrm{keV}$ and the discovery of six X-ray sources in that region, including a new black hole candidate close to GX 5-1; variability was detected in a one arcmin region around SgrA in the 3-20 keV band. The source has a very low luminosity $\left(-2 \times 10^{36} \mathrm{erg} / \mathrm{sec}\right)$ which is hundreds of millions of times below the Eddington luminosity for a $10^{6} \mathrm{M}_{0}$ black hole.

Mir-Kvant: Broad band 2-400 keV spectra were obtained for five X-ray Novae (GS 2023+38, Nova Vul, Nova Per, Nova Oph 1993. Nova Vel 1993); prominent soft components and extremely hard X-ray tails were detected; during six years of imaging of the central part of our Galaxy nine new X-ray sources were detected.

ROSAT: Soft X-ray spectra of galactic binaries, $26 \mathrm{X}$-ray sources identified with the globular clusters in M31.

Ginga: Broad band spectra and time variability of galactic X-ray sources.

\section{SUPERNOVA REMNANTS}

ROSAT: Detection of X-rays from 56 previously known galactic radio SNRs during the all-sky survey; detection of X-rays from about 90 sources suggestive of new, radio-uncatalogued SNR candidates; three candidates have been confirmed as SNRs up to now, fist full size mapping with few arcmin resolution of the giant SNRs like the North Polar Spur and other giant radio loops; spatial and spectral mapping of the medium size remnants revealing strong brightness and temperature variations; detection of a shell of cold matter associated with the Vela SNR casting an absorption shadow across Puppis-A; detection of EUV emission from the X-ray bright portions of the Vela SNR, high resolution mapping of the Crab Nebula revealing enhanced $X$-ray emission from an inner toroidal region and from two opposite jet-like features apparently protruding from the pulsar. detection of $X$-rays from the region containing the highest velocity optical knots at the very outskirts of Cas-A (Aschenbach, 1993)

BBRXT: High resolution, broad band spectra $(0.5-10 \mathrm{keV})$ of 7 galactic SNRs and 2 SNRs in the Large Magellanic Cloud; spectra of Cas-A and Tycho indicate the need for multiple non-equilibrium emission models.

ASCA: High resolution, broad band CCD X-ray spectra $(0.5-10 \mathrm{keV})$ of galactic and extragalactic SNRs exhibiting an unprecedented wealth of emission lines. 


\section{SUPERNOVAE}

ROSAT: First detection of soft X-rays from SN 1987A in 1991 (Beuermann et al, 1993); first detection of soft X-rays from SN 1993 J in M8I as early as 6 days after the supernova explosion and measurement of the $\mathrm{X}$-ray light-curve for the first 40 days (Zimmermann et al.,1993). Measurements were resumed on November 1, 1993, and soft X-rays have been detected again.

ASCA: Early measurement of the broad-band X-ray spectrum of SN $1993 \mathrm{~J}$; measurement of red and blue Doppler-shifted X-ray emission lines from the jets of SS 433 .

\section{DIFFUSE GALACTIC X-RAY EMISSION}

Granat: A map and the spectrum of the galactic center X-ray diffuse source was obtained. It was found that SgrA was weak in $X$-rays during the past 400 years

ROSAT: Discovery of X-ray-shadowing by dense neutral clouds; exploration of the spatial distribution of hot and cold galactic gas (Snowdon et al., 1992 ); strong evidence for the existence of a $10^{6} \mathrm{~K}$ hot galactic halo; discovery of soft X-ray emission from hot superbubbles. large evolved supernova remnants and HII regions (e.g. Loop I. Eridanus Enhancement, Orion-Monoceros Complex, Ophiuchus Region).

\section{NEARBY GALAXIES}

ROSAT Survey: 450 optically catalogued galaxies detected, high X-ray luminosity spirals found by correlations with the IRAS catalogue (Pietsch and Trümper 1992).

ROSAT: Mapping the diffuse emission of LMC. SMC: discovery of supersoft X-ray sources in LMC, SMC, M31 and other galaxies: discovery of $\sim 400 \mathrm{X}$-ray sources in M31: discovery of soft X-ray halos in NGC 253, M101, NGC 891. NGC 4631. NGC 4565. NGC 4258.

\section{ACTIVE GALACTIC NUCLEI}

ROSAT: Large number $(\approx 20000)$ of X-ray detected AGN; cross correlation with radio surveys yield 2500 mostly optically unidentidied radio loud AGN: confirmation of soft X-ray excesses in Seyfert $I$ and QSO spectra ; tight connection between soft X-ray excesses and the big blue bump in AGN spectra (Walter et al. 1993); detection of extremc X-ray luminosities in starburst galaxies; X-ray detection of high redshift quasars up to $Z=4.32$. detection of rapid $X$-ray variability $(\sim 800 \mathrm{~s})$ in narrow line Syl galaxies (e.g.IR 310224-3809), nearly simultancous optical and X-ray variability of the BL Lac object PKS 2155-304; absence of periodicity in NGC 6814 (the claimed 12800 s period is due to a nearby CV, Madejski et al.,1993).

Ginga: Confirmation of reflection/warm absorption models for AGN (Pounds et al., 1990).

ASCA: Line diagnostics confirms iron lines/warm absorber models for several AGN.

Granat: Detection of a thermal cut-off in the X-ray spectrum of NGC 4151 . 


\section{CLUSTERS OF GALAXIES}

Einstein and EXOSAT observations indicate a steepening of the cluster X-ray luminosity function, i.e. a deficit of luminous clusters at high redshift. This is consistent with hierarchical theories of cluster formation, in which merging processes play a central role. Early ROSAT results support the Einstein and EXOSAT findings.

Ginga obtained spectra of about 100 clusters. An analysis of the data indicates that the hotter, more massive and more luminous clusters contain relatively more gas and relatively less iron, so that the proportion of primordial gas is higher.

ROSAT observations show sub-structure in about $70 \%$ of clusters, suggesting that mergers are frequent, consistent with hierarchical theories of cluster formation. A comparison of radio and ROSAT images of clusters reveals irregular X-ray emission near the radio position in about $75 \%$ of the cases, consistent with the cause being a merger event (Henry and Briel 1993)

ROSAT observations of small compact galaxy (Hickson) groups show them to be rapidly evolving systems with massive haloes of dark matter.

ROSAT observations affirm the hypothesis that most clusters contain cooling flows, which result in a steadily increasing mass of cold dark clouds accunulating in the cluster core.

The radial distribution of dark and baryonic matter was measured using ROSAT cluster observations (A2256, A426 Coma, Perseus, Virgo clusters). The results of such observations should enable eventually constraints on the matter density in the universe to be established (Böhringer, 1993).

Results from the ROSAT survey of clusters yields the first three-dimensional spatial correlation function for an X-ray selected sample of clusters. The result is important for studies of the growth of matter fluctuations and galaxy formation in an evolving universe.

\section{COSMOLOGICAL X-RAY BACKGROUND}

COBE rejects the hot intergalactic medium hypothesis for the extragalactic X-ray background, (Mather et al., 1990).

Ginga shows that AGN spectra are not canonic power laws (Pounds et al., 1990).

Ginga and HEAO-1 fluctuation analyses find a factor 2-3 higher source counts than the Einstein Medium Sensitivity Survey, indicating the importance of intrinsic absorption.

ROSAT Deep Surveys reach a sensitivity more than a factor of 10 fainter than the deepest Einstein pointings and resolve more than $75 \%$ of the $1-2 \mathrm{keV}$ background into discrete objects/fluctuations (Hasinger et al., 1993).

Optical Identifications in ROSAT deep fields are mainly AGN, a substantial fraction optically fainter than the limiting capabilities of current $4 \mathrm{~m}$-class telescopes (Shanks et al., 1991). 
References:

Aschenbach, B., 1993, Advances in Space Research, in press

Becker, W., and Trümper, J., 1993, Nature $\underline{365}, 528$

Beuermann, K. et al., 1993, Astron Astroph. 281, L45

Böhringer, H., 1993, Advances in Space Research, in press

Bunk, W., and Verbunt, F., 1994 to be published

Doyle, J. et al., 1991, MNRAS 248,503

Halpern, J., and Holt, S.. 1992, Nature 357. 222

Hasinger, G. et al., 1993, Astron Astroph. 275, 1

Henry, J.P., and Briel, 1993, Advances in Space Research, in press

Madejski, G.M. et al., 1993, Nature 365, 626

Mather, J.C. et al., 1990, Ap.J. (Lett.) 354, L37

Pietsch, W., and Trümper, J., 1993, Advances in Space Research, in press

Pounds, K., 1990, Nature 344, 132

Schmitt, J.H.M.M., 1992 in "Solar and Stellar Cornoae" ed P. Pallavicini in Mem. Soc. Astr. It. 63, 563

Schmitt, J.H.M.M., 1994, Ap.J., Supp., in press

Shanks, T. et al 1991, Nature $3.53,315$

Snowden, S. et al., 1992, 179th American Astron.Soc. Meeting

Walter, R. et al., 1993. Astron.Astroph. 274. 105

Zimmermann, H.K. ct al.. 1993. Nature in press 


\section{Gamma-Ray Astronomy}

Volker Schönfeider

In gamma-ray astronomy we are presently experiencing a period of exciting new results from the Compton Gamma-Ray Observatory of NASA. Compton was launched on April 5, 1991 by the Space Shuttle Atlantis and is since then extremely successful. It is the first satellite mission that covers the full space astronomy gamma-ray range from about $50 \mathrm{keV}$ to $30 \mathrm{GeV}$ - more than five orders of magnitude in photon energy. Compton contains four different instruments with complementary properties to cover the broad spectral range (see Schönfelder, 1990). These are:

OSSE (50 keV to $10 \mathrm{MeV}$ ), COMPTEL (0.75 MeV to $30 \mathrm{MeV}$ ), EGRET ( $30 \mathrm{MeV}$ to $30 \mathrm{GEV}$ ), and BATSE (50 keV to $10 \mathrm{MeV}$ ).

Compton has started its mission with an 18-month lasting full sky survey - the first one in gamma-ray astronomy at all. Since November 1992 extended observations of selected sky regions or targets are being performed. Most of the data from the survey phase have already been released for public access, and a vigorous Guest Investigator Program is now being performed.

A number of exciting discoveries has been made by Compton so far: the distribution of gammaray bursts on the sky - as measured by BATSE - has disproofed the previous widely accepted neutron star origin of bursts within the galactic disk (Meegan et al., 1992). Instead, solar system, galactic halo and cosmological origins are now being discussed. The puzzle about the origin of the burst has become more challenging than ever (Fishman, 1993).

The discovery of gamma-ray blazars by EGRET was a surprise. More than 30 of these objects have now been detected. Their high-energy gamma-ray emission seems to be produced in jets. In most cases, the luminosity at gamma-ray energies is higher than in any other part of the electromagnetic spectrum (Fichtel et al., 1993).

Also radio pulsars seem to have their peak-luminosity at gamma-ray energies. In addition to the previously known two gamma-ray pulsars Crab and Vela, pulsed gamma-ray emission has now been detected from PSR 1509-58, 1706-44, 1055-52 and Geminga, as well. Geminga may represent a new class of pulsars. It is only seen at $x$ - and $y$-ray energies, but not in the radio band. It is interesting to note that the efficiency for converting rotational energy into gamma-ray luminosity increases with the age of the pulsar (Harding, 1993). 
Much progress has been achieved in the field of gamma-ray line spectroscopy: COMPTEL has generated the first complete map of the galactic plane in the light of the $1.8 \mathrm{MeV}{ }^{26} \mathrm{Al}-$ line. The distribution along the plane is not smooth, but contains bright spots, which might suggest a more local origin (Diehl et al, 1993). One of the spots coincides with the Vela supernova remnant, and the detected line flux is well within the expectations from a Type-II Supernova. Two other nucleosynthesis lines were detected for the first time: these are the $122 \mathrm{keV}$ line from ${ }^{57} \mathrm{Co}$ by OSSE (from SN 1987A), and the $1.156 \mathrm{MeV}$ from ${ }^{44} \mathrm{Ti}$ by COMPTEL (from Cas A). The detection of $4.4 \mathrm{MeV}$ and $6.1 \mathrm{MeV}$ line emission from the Orion complex by COMPTEL marks the first measurement of nuclear interaction lines from interstellar space. OSSE, at present, is sampling a composite image of the inner part of the galaxy in the light of the $511 \mathrm{keV}$ annihilation line. This image looks significantly different from the $1.8 \mathrm{MeV}{ }^{26} \mathrm{Al}$-image.

These highlights represent only a small sample of the Compton results. Investigations of a large number of $\mathrm{x}$-ray binaries and transients, of the diffuse galactic gamma-ray emission and of several spectacular solar flares, are only mentioned here. The analysis of the Compton data is far from being completed and many more results are still to be expected.

\section{References:}

R. Diehl et al., Adv.Sp.Res. $\underline{13}$, No 12, 723-726 (1993)

C. Fichtel et al., AIP-Proc. $280,461-472$ (1993)

J. Fishman, AIP-Proc. 280, 669-680 (1993)

A. Harding, Proc. of 16th Texas-Symposium (Berkeley, Dec 1992)

C. Meegan et al., Nature $\underline{355}, 143$ (1992)

V. Schönfelder, Adv.Sp.Res. 10, No 2, 243-253 (1990) 
6. Solar Physics from Space 1990-1993

Stuart D. Jordan

The period covered by this report was dominated by three developments: (1) a continuing study of the maximum phase of Solar Cycle 22, especially through the successful launch and operation of the YOHKOH mission by the Institute of space and Astronautical Sciences, (ISAS) of Japan; (2) the continuing development of instruments and spacecraft for the solar and Heliospheric Observatory (SoHO), a joint mission of the European Space Agency (ESA) and the American National Aeronautics and Space Administration (NASA); (3) the continuing, but so far unsuccessful, attempt of the international solar commuity to develop a 1-meter class optical telescope for operation in space. These and a few other developments are reviewed here.

The most significant event for solar physics from space during the period covered by this report was the successful launch and early operation of the YOHROH satellite by ISAS. Launch occurred on 30 August 1991 from the Kagoshima space Center, and operation to date has yielded a large database for the study of the highenergy solar phenomena that accompany solar activity. The satellite carries four major instruments: a hard $\mathrm{x}$-ray telescope (HXT), a soft X-ray telescope (SXT), a set of wide band spectrometers (WBS), and a set of Bragg crystal spectrometers. The spacecraft and much of the instrument development were accomplished in Japan, where the mission operations control center is located. There was also substantial participation in instrument development in the U.K. and in the U.S.A. The ISAS ground station at Kagoshima and the NASA Deep Space Network provide the communications link with the sateliite, which has an on-board bubble memory with an $80 \mathrm{Mb}$ capacity. Excellent references for a more detailed description of all aspects of the mission, scientific as well as technical and programmatic, appear in Solar Physics, Volume 136 (all papers), and in Publications of the Astronomical Society of Japan, Volume 44, No. 5 (all papers).

The HXT on YOHKOH was designed to obtain observations of hard Xrays from solar flares with an unprecedented combination of angular, temporal, and energy resolution, in order to better study the process of electron bremsstrahlung in flares. From observations with an angular resolution of 5 arcseconds and a temporal resolution of 0.5 seconds, taken simultaneously in the four energy bands 14-23-33-53-93 kev, it has become possible to study in far more detail than was previously possible the generation and confinement of the high energy electrons known to be associated with flares. HXT data have already been obtained on hundreds of flares.

The SXT on YOHKOH has already obtained on the order of one million excellent soft $x$-ray pictures of the sun from which 
future studies of solar active regions and flares will benefit. The soft $X$-ray regime is thought to be the primary energy range for radiative losses in many strong active regions and flares, so high resolution observations are essential for understanding the energy balance in coronal loops and the energy transfer process in flares.

The WBS instrument has already provided evidence for two types of gamma-ray flares, for significant time variations of the hard $x-$ ray spectrum that will provided further insight into the acceleration of solar flare electrons, and for proton acceleration in some of the flares observed. This instrument provides Iight-curve observations in ten energy bands with a temporal resolution in the range 0.125-0.5 seconds, as well as energy spectra from three spectrometers with temporal resolution 1-4 seconds, over a broad energy range from $3 \mathrm{keV}$ to $100 \mathrm{MeV}$.

Finally, the BCS is gathering data to address questions of plasma heating and dynamics during the impulsive phase of solar flares. The BCS observes the sun in four wavelength bands between $1.7 \mathrm{~A}$ and $5.1 \mathrm{~A}$. Altogether, the four instruments on YOHKOH have already yielded a database that will keep high-energy solar physicists busy for the entire decade, as they attempt to resolve some of the still outstanding problems of solar flare physics: mechanisms for the acceleration of electron beams, the presence and acceleration of proton beams, nonthermal versus thermal models for (perhaps different types of) flares, what are the major types of flares (more refined classification, as a function of dominant physical processes), and further constraining of energetic processes, perhaps leading to improved ideas on different classes of flare "trigger" mechanisms.

In parallel with this major mission, solar flare research continues on older data sets obtained largely from the now defunct Solar Maximum Mission (SMM) and Hinatori. When all of the space data on solar flares and solar activity have been combined with an equally impressive database obtained from the ground, especially in the radio regime (Dennis and Zarro, 1993), we can expect significant advances in our understanding of solar flares by the end of the decade.

The other major solar space mission that has been the focus of attention during the period 1991-1993 is the soHo mission, a mission dedicated to the better understanding of the solar corona and the solar interior. SoHo remains scheduled for launch in the late summer of 1995. At the time of this writing the soHo instruments are under final development, to be delivered during 1994 for integration into the spacecraft. If all goes as planned, the mission will operate for two or more years, collecting data from the first Lagrangian point between the Earth and the sun where continuous solar viewing will be possible. A comprehensive summary of the mission, its instruments, and its scientific objectives appears in ESA SP-1104 (1989). 
Major problems in coronal physics will be attacked with these data. The "Parker" mechanism of solar wind acceleration by the thermal pressure gradient can produce supersonic expansion of the wind, and come close to predicting some of the wind properties over the quiet Sun, but this mechanism, alone, is inadequate to explain solar-wind acceleration in the high-speed streams now known to be associated with coronal holes. Two coronagraphs on SoHO will attempt to observe the wind out to at least 10 solar radii, in order to better understand this acceleration process.

While it is understood that a hot corona will produce a negative thermal pressure gradient beyond some distance frum the sun, and thus contribute to solar wind acceleration, solar physicists still do not know what produces (i.e.) heats the corona in the first place, although theories abound. In order to provide a better observational base for evaluating theories of coronal heating, SoHO will carry two spectrographs which, together, will provide diagnostic spectra of high spatial and spectral resolution over a broad range of coronal and transition region temperatures and densities. This superior diagnostic capability will help to refine models of these solar regions, and further constrain theories on the underlying physics. A fifth instrument will provide EUV images of the sun, to provide context and additional science data. Other instruments will measure particle concentrations in the solar wind.

The other major goal of SoHo is to provide a database that, in conjunction with data from a developing ground-based solar network (developed by the Global Oscillations Network Group, or GONG) will permit solar physicists to map the density, temperature, and velocity distribution of the solar plasma from the photosphere down to the center of the sun. One instrument will observe full sun oscillations with very high sensitivity, concentrating on the lowest frequencies that penetrate to the solar core. Another instrument, a Michelson Doppler interferometer, will measure line-of-sight velocities and magnetic fields to high angular resolution ( 1.4 and 4 arcseconds) as well as other parameters, to provide improved models of the more superficial layers of the sun. A third instrument will measure variations in the solar irradiance on time scales of minutes up to the mission time to gain further insight into the very low frequency global oscillations.

If the news on YOHKOH is good, and on soHo hopeful, it must be reported that the news on the efforts of the solar community to achieve a l-meter class observing capability in space is bad. The hoped for orbiting Solar Laboratory (OSL), planned during the $1980^{\prime}$ 's by NASA, has been long delayed by budgetary constraints, and while it remains in the NASA long-range plan, most solar physicists are exploring other ways to achieve this capability sooner, if that is possible. Solar physicists in the United States have been invited by NASA to produce a plan for a solar Research Base Enhancement program that could include a balloon bourn telescope of large primary diameter approaching 1-meter, 
but it is not yet clear whether this program will materialize. The ISAS in Japan is exploring the possibility of building a large aperture, possibly $80 \mathrm{~cm}$, solar telescope (Solar-B) to be flown in space, though this remains in the planning stage as well at this writing.

Continual improvements in solar ground-based capability are partially closing the gap between the 0.1 arcsecond angular resolution goal possible from space and what can be achieved from the ground. Nevertheless, this writer is aware of no solar physicist who believes that 0.1 arcsecond angular resolution can be achieved from the ground with reasonable image stability over several minutes and over a field-of-view large enough to attack the fundamental problems of the role of the small-scale magnetic fields in the dynamics of the lower atmosphere. This very high angular resolution is necessary if we are to observe these dynamic atmospheric phenomena on the main scale of energy transfer in the photosphere and chromosphere, i.e., the atmospheric scale height and a continuum mean-free-path. The scientific need for this space-based facility remains.

In addition to the three areas of major activity mentioned, there are a number of other developments that could produce exciting new science over the rest of the decade. The first of the CORONAS missions is currently scheduled for launch in December of 1993, although there is a good chance that actual launch may occur in 1994, as Russia and Ukraine, the joint developers of the mission, restructure their joint space effort. This coRoNAs mission will be directed to general studies of the physics of the outer solar atmosphere, will carry a $200 \mathrm{~kg}$ scientific payload, and is scheduled to perform EUV spectroscopy and white light coronagraphy from low-Earth orbit, as well as carrying a highenergy payload for the study of solar activity (Sagdeev, 1993).

The Ulysses mission of ESA and NASA, with an international payload, is also expected to provide exciting new insights into the solar atmosphere, by directly observing for the first time the Sun's high latitude regions. The pass nearly over the southern pole of the sun is expected to occur during september of 1994 , to be followed by a pass at about 80 degrees north solar latitude during August of 1995. Science investigations include in situ probing of the solar wind as the spacecraft moves in toward the Sun on its "boomerang" orbit from Jupiter, as well as detailed observations of the high latitude regions of the solar atmosphere by nine instruments during the high latitude passes. Phenomena ranging from "superspicules" to polar coronal holes will be observed directly, and insight should be gained into the high latitude components of the solar wind.

Another option for operating science payloads in space was implemented successfully with the deployment from a NASA shuttle in April 1993 of a SPARTAN payload consisting of an ultraviolet coronagraph and a white light coronagraph. The SPARTAN is a completely autonomous subsatellite which, in this case, operated 
for 47 hours before recovery by the shuttle. Preliminary results and coronal images were presented at the Solar Physics Divisional Meeting of the American Astronomical Society in July 1993 (not published) and should appear in the open literature around the end of 1993.

With more fine data expected from Yohkoh, a second, upcoming flight of Spartan, as well as Coronas and Ulysses, there is considerable excitement in the solar community over an organized observing campaign that could involve all four missions.

Technical development and application continues in at least three areas of importance to solar physics in space. The first is the switch to a new family of two-dimensional digital detectors, many built around microchannel plates and charge-coupled devices (CCDs). Continuing development of these detectors on sounding rockets, and further use on major space missions such as SoHo will continue. The second major technical development is the use of new thin-film coatings on normal incidence telescopes, coatings that significantly increase the reflectivity of a normal incidence surface at EUV wavelengths, at least over a limited wavelength range. This in turn makes possible the construction of normal incidence telescopes in this range, capable of achieving higher angular resolution than has proved possible with grazing incidence designs. Finally, the increased application of sophisticated computer networks and data storage technicues is making data access from major space missions increasingly available to users worldwide, a development that can only further the already highly international, global character of solar astronomy.

\section{References}

Dennis, B.R., \& Zarro, D.M. 1993, Solar Physics, 146, 177.

Pub. Astron. Soc. Japan, 1992, 44, No. 5 (entire issue).

Sagdeev, R. 1993 (private communication).

Solar Physics, 1991, 136 (entire volume).

The Soho Mission, 1989, ESA SP-1104. 